\title{
Collective sparse symmetric non-negative matrix factorization for identifying overlapping communities in resting-state brain functional networks
}

\author{
Xuan Li ${ }^{\mathrm{a}, \mathrm{b}}$, John Q. Gan ${ }^{\mathrm{a}, \mathrm{b}}$, Haixian Wang ${ }^{\mathrm{a}, *}$ \\ ${ }^{a}$ Key Laboratory of Child Development and Learning Science of Ministry of Education, School of Biological Science E Medical Engineering, \\ Southeast University, Nanjing, Jiangsu 210096, PR China \\ ${ }^{b}$ School of Computer Science and Electronic Engineering, University of Essex, Colchester CO4 $3 S Q$, UK
}

\begin{abstract}
Resting-state functional magnetic resonance imaging (rs-fMRI) provides a valuable tool to study spontaneous brain activity. Using rs-fMRI, researchers have extensively studied the organization of the brain functional network and found several consistent communities consisting of functionally connected but spatially separated brain regions across subjects. However, increasing evidence in many disciplines has shown that most realistic complex networks have overlapping community structure. Only recently has the overlapping community structure drawn increasing interest in the domain of brain network studies. Another issue is that the inter-subject variability is often not directly reflected in the process of community detection at the group level. In this paper, we propose a novel method called collective sparse symmetric non-negative matrix factorization (cssNMF) to address these issues. The cssNMF approach identifies the group-level overlapping communities across subjects and in the meantime preserves the information of individual variation in brain functional network organization. To comprehensively validate cssNMF, a simulated fMRI dataset with ground-truth, a real rs-fMRI dataset with two repeated sessions and another different real rs-fMRI dataset have been used for performance comparison in the experiment. Experimental results show that the proposed cssNMF method accurately and stably identifies group-level overlapping communities across subjects as well as individual differences in network organization with neurophysiologically meaningful interpretations. This research extends our understanding of the common underlying community structures and individual differences in community strengths in brain functional network organization.
\end{abstract}

Keywords: non-negative matrix factorization, overlapping communities, resting state networks, inter-subject variability, test-retest reliability, resting state fMRI

\section{Introduction}

The human brain is a complex and delicate system, where distinct brain regions work in coordination to accomplish diverse neural functions. The recent decade has seen grow5 ing interests in introducing the concept of network into the field of neuroscience for studying brain system (Bullmore and Sporns, 2009). From the network viewpoint, brain ${ }_{20}$ networks (or graphs) can be simplified as sets of discrete neural elements (nodes) and their interactions (edges) for both structural and functional networks. Such network perspective opens a new avenue for investigating brain architecture and function by providing powerful analysis

\footnotetext{
* Corresponding author

Email address: hxwang@seu.edu.cn (Haixian Wang)
}

tools for brain imaging data. Moreover, the technology of functional magnetic resonance imaging (fMRI), especially resting state fMRI (rs-fMRI), provides a useful channel to study brain functional networks in depth. The rs-fMRI records the signals of spontaneous brain activities when no particular task is performed. The access to powerful network approaches and rich resources of brain imaging data has largely promoted studies on brain network organization (Sporns et al., 2004, Power et al., 2011, Bullmore and Sporns, 2012).

Two primary aspects in understanding brain network organization is the segregation and integration of brain functions. In particular, the functional segregation in brain networks is captured by identifying underlying communities, where a community (or module) consists of highly 
functionally-connected brain regions (nodes) that show coherent signal fluctuations. The communities also cor(RSNs), when resting-state fMRI data is applied (Sporns 2013, 2014). Prior studies have discovered several consistent RSNs in normal brains, including default mode network (DMN), fronto-parietal, visual networks and so forth (Van den Heuvel and Pol, 2010, Yeo et al., 2011, Buckner et al. 2013). Besides, significant findings have shown that brain disorders such as Alzheimer's disease (AD) and schizophrenia are related to alterations in network topology or in connectivity strengths of RSNs (Greicius et al. 2004, 40 von dem Hagen et al. 2012). Thus, studying RSNs can facilitate the understanding of not only the organization and functions of normal brains but also the pathological process of neurological illness based on brain imaging data.

Identification of RSNs (or network communities) is of great importance. In an early work, RSNs were constructed 90 based on the correlations between seed regions of interest (ROIs) and other nodes (Greicius et al. 2003). Recently, researchers have resorted to various techniques to identify the RSNs distributed over the whole brain. According to (Sporns, 2014), these methods mainly fall into the follow-95 ing groups: network-based community detection methods (Power et al., 2011), clustering analysis (Yeo et al., 2011) and independent component analysis (ICA)(De Luca et al. 2006; Smith et al., 2012).

${ }_{55}$ In computational neuroscience, identifying network com-100 munities by clustering highly functionally-connected regions is termed as community detection. Several resting-state fMRI studies have applied community detection methods to the whole-brain network (Power et al. 2011). The wholebrain network is typically constructed by taking disjoint ${ }_{105}$ brain regions as nodes and temporal dependency of their time series (i.e., functional connectivity) as edges (Biswal et al. 1995). In fact, clustering methods are very similar to the community detection methods, which group coherent regions into different clusters based on the affinity (usually ${ }_{110}$ measured by functional connectivity) between these regions (Van den Heuvel and Pol, 2010; Yeo et al., 2011). For these two groups of methods, a major disadvantage is that most of them typically identify a non-overlapping community

70 structure under the assumption that one node will be as-115 signed to one community/cluster only and few methods concern the overlapping community structure. However, accumulating evidence has shown that the brain network, like many other kinds of realistic complex networks, actually has an overlapping community structure in the sense that one brain region participates in multiple communities (O'Reilly et al., 2010, Cole et al., 2013, Pessoa, 2014). Although several efforts have been made on this critical issue in both structural (Wu et al., 2011) and functional brain networks Smith et al. 2012, Eavani et al. 2015, Najafi et al., 2016), the overlapping community structure of brain networks are still largely unclear.

In contrast, ICA differs significantly from the community detection and clustering methods. Instead of using the functional connectivity between regions, ICA factorizes the data matrix of fMRI time series directly into components that achieve maximal spatial or temporal independence (spatial ICA (sICA) and temporal ICA (tICA) respectively). Since the assumptions are made in different situations, the components (or RSNs) derived by sICA and tICA are termed as intrinsic connectivity networks (ICNs) and temporal functional modes (TFMs) respectively. As a decomposition method, there is no constraint to the strengths of each region across components, and thus ICA results in overlaps between different components. However, ICA makes strong assumptions about the statistical dependency of components. A major drawback is that it tends to produce dense components with negative values, which lack direct and explicit interpretation both physically and physiologically.

Another common issue of the aforementioned methods is that the group-level community structure across subjects is often identified by collapsing the fMRI data of multiple subjects. And during this process, the inter-subject variability in community structure is typically ignored or not directly reflected. Additional steps are thus needed to analyze these individual differences. Actually, the intersubject variability provides valuable resources especially for the discrimination of different groups (e.g., normals and patients) or for the identification of individuals (Finn et al. 2015). Significant alterations have been found in functional connectivity strength and network topological measures in the brain with diseases like AD Greicius et al. 2004). It is suggested that the inter-subject variability is reflected in community strengths (see Equations (4) and (5) for definition), indicating the extent to which a specific community is involved in the global functional network of a subject (von dem Hagen et al., 2012). However, only a 
few studies have taken this into consideration and depicted such inter-subject variability quantifiably without postprocessing steps when developing computational models165 (Eavani et al. 2015).

Recently, a useful technique, non-negative matrix factorization (NMF), has drawn great attention in fMRI studies. NMF was first proposed by Lee and Seung (1999) and has widely used in the domain of image processing and text mining. Several studies have applied NMF to fMRI data (Wang et al., 2004, Potluru and Calhoun, 2008; Ferdowsi et al. 2010,2011 . Given a data matrix $\mathrm{X} \in \mathbb{R}^{d \times n}$ containing $d$ time points and $n$ brain ROIs/voxels, NMF approximately factorizes $\mathrm{X}$ into the product of $\mathrm{B} \in \mathbb{R}^{d \times k}$ and $^{175}$ $\mathrm{H} \in \mathbb{R}^{k \times n}$ (i.e., $\mathrm{X} \approx \mathrm{BH}$ ), where $k$ is the reduced rank. As can be seen, NMF is closely related to ICA, since they both seek a representation of the original data by using the linear combination of bases with a lower rank $k$. However, unlike allows negative weights in the components, NMF allows only additive combinations by enforcing non-negativity constraints on both B and H. Hence, NMF learns a part-based representation by naturally identifying coherent parts to form the whole with only additive combinations (Lee and ${ }^{85}$ Seung, 1999). Although NMF has manifested the ability to perform clustering effectively, it may not be a very suitable approach to identifying communities or clusters. Firstly, the primary goal of NMF is to perform dimensionality reduction by approximating data with fewer bases, rather ${ }^{190}$ than to partition the data points into coherent clusters based on their interrelations (Kuang et al. 2012). Secondly, the non-negativity of NMF suppresses the negative values in fMRI signals of the input data matrix, however, these negative signals might be physiologically meaningful (Xie et al., 2017). A better alternative to NMF for performing clustering is one of its variants, symmetric NMF, which has been recently used in community detection in fields of data mining (Ding et al., 2005; Wang et al., 2011).

By contrast, the symmetric NMF decomposes an affinity matrix $G$ which measures the affinity between each pair of data points into a cluster membership matrix $\mathrm{H}$ of size $n \times k$ as $\mathrm{G} \approx \mathrm{HH}^{\mathrm{T}}$. In our previous work, we have applied the symmetric NMF to detect the overlapping community structure at the individual level on fMRI data and the method has achieved good performance (Li et al., 2016a b).

Inspired by the symmetric NMF, in this paper, we pro- pose a collective symmetric sparse non-negative matrix factorization (cssNMF) method. The proposed cssNMF inherits the soft clustering effect from symmetric NMF, which factorizes any non-negative symmetric affinity matrix (not necessarily positive definite) into overlapping clusters, allowing one part to participate in multiple clusters. Moreover, we enforce sparsity on cssNMF by adding a sparsity constraint in the form of $\ell_{1}$-norm, as used in the sparse NMF (Hoyer, 2002). Although the symmetric NMF, like other NMF methods, has an accompanying effect that encourages sparsity automatically, the trade-off between sparsity and reconstruction error cannot be controlled explicitly. It has been suggested that algorithms enforcing sparsity may better capture the underlying structure of data and generate results with a better interpretation of the physical mechanism (Eavani et al., 2015, Xie et al., 2017). Therefore, in theory, the proposed cssNMF is expected to discover a sparse and overlapping brain community structure with a more straightforward interpretation. Like the symmetric NMF, cssNMF also operates on affinity matrices. In this paper, we introduce a collective generalization into cssNMF to identify the group-level community structure across subjects by taking as input the affinity matrices of all subjects simultaneously, instead of the commonly used group-averaged one. Furthermore, in order to capture the inter-subject variability, we adopt a weighted form of symmetric NMF by incorporating a weight matrix (Ding et al. 2005). This formulation gives the communities extra degree of freedom and makes space for preserving the individual variability in community strengths.

It should be noted that an accurate construction of the affinity matrix is essential for community detection. In fMRI studies, the affinity matrix is typically calculated by using the most widely-used Pearson correlation, which corresponds to an association matrix representing the wholebrain network. However, the Pearson correlation method calculates only the pairwise associations between nodes without ruling out the influence of other nodes and produces a dense association matrix with negative values that are difficult to interpret. Recently, a bunch of sparse representation based approaches has been widely applied to the construction of brain network (Huang et al., 2010; Wee et al. 2014, Li and Wang, 2015). These approaches obtain the associations between one node and all the other nodes simultaneously and retain only a few important associa- 
tions, based on the idea that the brain network is thought to be sparse (Fransson, 2005; Sporns, 2010). In this paper, we mainly apply cssNMF to association matrices constructed by non-negative adaptive sparse representation (NASR), which was proposed and applied in our previous work and has shown great performance (Li et al., 2016a b).

To validate the proposed cssNMF, it is compared with wo state-of-the-art network-based community detection methods, Infomap and modularity optimization, and two ICA methods, i.e., sICA and tICA on both real and simulated datasets. To quantitatively measure their ability in community detection, we first apply these methods on a simulated fMRI dataset with a ground-truth of the underlying network configuration. Then real resting-state fMRI data are used to mainly evaluate the reproducibility and test-retest reliability (Zuo et al. 2010). Furthermore, besides NASR, we also conduct the experiment based on the association matrices constructed by the Pearson correlation on simulated data for all network-based community detection methods including cssNMF, Infomap and modularity optimization. To sum up, cssNMF aims to serve the following two main purposes. Firstly, it identifies the groupevel overlapping community structure across subjects with a straightforward interpretation. Secondly, it meanwhile retains the inter-subject variability in community strengths without additional post-processing steps.

The rest of this paper is organized as follows. In Section 2 , we elaborate the proposed cssNMF and describe experimental settings. Section 3 presents the experimental results on both simulated and real fMRI datasets. Discussion and conclusion are given in Section 4 and Section 5 respectively.

\section{Materials and Methods}

Before applying cssNMF to identifying communities, an association matrix for each individual is constructed to capture the affinity between brain network nodes. Below, we briefly introduce the NASR method used in this work.

\subsection{Constructing association matrix by NASR}

Nodes of the brain functional network are usually represented as ROIs of the brain cortex, which can be derived based on a predefined atlas or by data-driven analysis. Once the network nodes are defined, the associations of these nodes are then measured by NASR using their fMRI time series. Specifically, let the normalized fMRI time series of $n$ nodes with $d$ time-points be denoted as $\mathrm{X}=\left(\mathrm{x}_{1}, \ldots, \mathrm{x}_{n}\right) \in \mathbb{R}^{d \times n}$, where sample $\mathrm{x}_{i}$ is the fMRI time series of the $i$ th node. In the NASR problem, $\mathrm{x}_{i}$ is approximately represented by its corresponding dictionary $\mathrm{X}_{i}=\left(\mathrm{x}_{1}, \ldots, \mathrm{x}_{i-1}, \mathrm{x}_{i+1}, \ldots, \mathrm{x}_{n}\right) \in \mathbb{R}^{d \times(n-1)}$ containing all samples except for $\mathrm{x}_{i}$ itself with a coding vector $w_{i} \in \mathbb{R}^{n-1}$ that is constrained to be non-negative and sparse. It is formulated as follows (Li et al. 2016a):

$$
\min _{w \geq 0} \frac{1}{2}\left\|\mathrm{x}_{i}-\mathrm{X}_{i} w_{i}\right\|_{2}^{2}+\lambda\left\|\mathrm{X}_{i} \operatorname{Diag}\left(w_{i}\right)\right\|_{*},
$$

where $\lambda>0$ is a regularization parameter. $\|\operatorname{XDiag}(w)\|_{*}$ is a trace least absolute shrinkage and selection operator (LASSO) regularizer which computes the sum of all singular values of $\operatorname{XDiag}(w)$, where $\operatorname{Diag}(w)$ represents a diagonal matrix with $w$ as its diagonal elements (Grave et al. 2011). It is the very feature of the trace LASSO regularizer that makes NASR stand out from other sparse representation based approaches. It is well-known that fMRI signals are likely to be highly correlated and such multicolinearity tends to cause failure for traditional LASSO sparse representation, since a sparse predictor will arbitrarily select one or several from these correlated variables, leading to an unstable situation. However, the trace LASSO regularizer adaptively makes a trade-off between the sparsity effect of $\ell_{1}$-norm and the grouping effect of $\ell_{2}$-norm, thus highly suitable to be applied on fMRI data. Specifically, when all samples $\mathrm{x}_{i}$ in $\mathrm{X}$ are orthogonal, the trace LASSO will behave the same as the $\ell_{1}$-norm, whereas when $\mathrm{x}_{i}$ are highly correlated (say identical in the extreme case), it is equal to the $\ell_{2}$-norm, as shown in Equation (2) (Grave et al. 2011

$$
\|w\|_{2} \leq\|\operatorname{XDiag}(w)\|_{*} \leq\|w\|_{1}
$$

Besides, the non-negative constraint on $w_{i}$ ensures that only the samples that positively contribute to the representation of $\mathrm{x}_{i}$ are likely to be selected, which leads to the results easy to interpret. For each $w_{i}$, the associations between node $i$ and all other nodes are obtained simultaneously by solving Equation (1) using alternating direction method (ADM) (Boyd et al., 2011), as used in Lu et al. (2013); Li and Wang (2015). The resulting $w_{i}$ is extended to $\tilde{w}_{i}$ by inserting a zero in its $i$ th position, indicating the association between node $i$ and itself is set to zero. Finally 
a symmetric association matrix is constructed by

$$
\mathrm{G}=\left(\mathrm{W}+\mathrm{W}^{\mathrm{T}}\right) / 2,
$$

where $\mathrm{W}=\left(\tilde{w}_{1}, \ldots, \tilde{w}_{n}\right) \in \mathbb{R}^{n \times n}$. In short, the association matrix constructed by NASR, which is non-negative and symmetric with a clear physical meaning, can directly be used as the input of cssNMF. Details of NASR and its early version, adaptive sparse representation (ASR), can be found in our previous works (c.f. Li and Wang, 2015 . Li et al., 2016a).

\subsection{Identifying community structure by cssNMF}

\subsubsection{Problem formulation}

Given the symmetric non-negative association matrices of M subjects, derived by NASR, $\mathrm{G}^{i} \in \mathbb{R}^{n \times n}(i=1, \ldots, M)$, cssNMF aims to factorize these matrices collectively into $k$ group-level communities $\mathrm{H}=\left(h_{1}, \ldots, h_{k}\right) \in \mathbb{R}^{n \times k}$ across subjects, where each column $h_{j}$ is the membership vector of the $j$ th community. Simultaneously, for each subject the individually specific information is retained in a vector $s^{i}=\left(s_{1}^{i}, s_{2}^{i}, \ldots, s_{k}^{i}\right) \in \mathbb{R}^{k}$, where the scalar $s_{j}^{i}>0$ indicates the strength of the $j$ th community of the $i$ th subject. In the model of cssNMF, it is represented by a diagonal weight matrix $\mathrm{S}^{i}=\operatorname{diag}\left(s^{i}\right) \in \mathbb{R}^{k \times k}$ with $s^{i}$ as its diagonal elements and zeros as off-diagonal elements. Mathematically, the proposed cssNMF method is formulated as

$$
\begin{aligned}
\min _{\mathrm{H}, \mathrm{S} \geq 0} & \frac{1}{2} \sum_{i=1}^{M}\left\|\mathrm{G}^{i}-\mathrm{HS}^{i} \mathrm{H}^{\mathrm{T}}\right\|_{\mathrm{F}}^{2}+\beta\|\mathrm{H}\|_{1} \\
\text { s.t. } & \forall j: \max \left(h_{j}\right)=1, j=1, \ldots, k,
\end{aligned}
$$

where $\mathrm{H}$ and $\mathrm{S}^{i}$ are restricted to be non-negative, $\|\cdot\|_{\mathrm{F}}$ denotes the Frobenius norm and the regularization parameter $\beta>0$ controls the sparsity level of the obtained communities. More specifically, the sparsity constraint $\|\mathrm{H}\|_{1}$ in the form of $\ell_{1}$-norm enforces the obtained communities to retain only the most relevant nodes by assigning them larger ${ }_{295}$ membership values and eliminate the less important nodes with membership values near zeros. The sparsity constraint improves the cssNMF method from two aspects. Firstly, it makes the cssNMF method more robust by reducing its risk of overfitting. Secondly, it helps to seek a better interpretation of the obtained community structure. Besides, in order to obtain the common community structures shared by multiple subjects, we factorize the association matrices of all subjects simultaneously and minimize the collective reconstruction error, instead of taking the commonly used group-averaged association matrix as input. It brings about a direct benefit that the individually specific information is retained. Based on this, we further introduce the diagonal weight matrix $S^{i}$ to preserve the inter-subject variabilities. Moreover, an important distinction between cssNMF and other matrix factorization methods is that no assumptions have been made on cssNMF except for the constraint of non-negativity. The non-negative constraint enables cssNMF to learn a part-based representation of the original data, thus enhancing the interpretability of the results. The constraint of the input association matrix is also relaxed, indicating that it is not necessary to be positive definite. Any association (affinity) matrix can be factorized by cssNMF as long as it is symmetric and non-negative.

In other words, cssNMF pursues an approximation of the association matrix for each individual by a non-negative linear combination of $k$ identified consistent communities across all individuals with the diagonal elements in $S^{i}$ as the corresponding combination coefficients. Further, the association matrices can be represented as

$$
\mathrm{G}^{i} \approx s_{1}^{i} h_{1} h_{1}^{\mathrm{T}}+s_{2}^{i} h_{2} h_{2}^{\mathrm{T}}+\ldots+s_{k}^{i} h_{k} h_{k}^{\mathrm{T}}
$$

${ }_{285}$ where $h_{j} h_{j}^{\mathrm{T}}$ denotes the $j$ th community and $s_{j}^{i}$ represents its strength. Note that the solution of this problem is not unique due to the arbitrary scales of $h_{j}$ and $s_{j}^{i}$, i.e., $s_{j}^{i} h_{j} h_{j}^{\mathrm{T}}=\left(s_{j}^{i} /\left(\alpha^{2}\right)\right)\left(\alpha h_{j}\right)\left(\alpha h_{j}^{\mathrm{T}}\right)$ where $\alpha$ is a scalar. For this reason, we restrict the maximum value of membership to each community to be unity.

\subsubsection{Algorithm for cssNMF}

The optimization of $\mathrm{S}^{i}$ is a convex problem. It could be solved by using the multiplicative algorithm (Lee and Seung, 2001), which is simple but efficient for solving NMF problems. However, the optimization of $\mathrm{H}$ is somehow complicated due to the non-convex constraint on $h_{j}$. We thus adopt the strategy of projected gradient descent to solve the problem, following the procedures in Hoyer (2002).

Specifically, by fixing $\mathrm{H}, \mathrm{S}^{i}$ is optimized by the multiplicative update rule used in (Ding et al. 2005):

$$
\mathrm{S}_{j l}^{i} \leftarrow \mathrm{S}_{j l}^{i} \frac{\left(\mathrm{H}^{\mathrm{T}} \mathrm{G}^{i} \mathrm{H}\right)_{j l}}{\left(\mathrm{H}^{\mathrm{T}} \mathrm{HS}^{i} \mathrm{H}^{\mathrm{T}} \mathrm{H}\right)_{j l}} .
$$


As long as $\mathrm{S}^{i}$ is strictly initialized as a non-negative diagonal matrix, the update rule in Equation (6) can ensure the non-negativity of the resulting $\mathrm{S}^{i}$ and the non-diagonal $\mathrm{l}_{320}$ elements are set to zero automatically. Then by fixing $\mathrm{S}^{i}$, $\mathrm{H}$ is updated by using the projected gradient descent:

$$
\mathrm{H} \leftarrow \mathrm{H}-\mu\left(\sum_{i=1}^{M} \mathrm{HS}^{i} \mathrm{H}^{\mathrm{T}} \mathrm{HS}^{i}-\mathrm{G}^{i} \mathrm{HS}^{i}+\beta\right),
$$

where $\mu$ is the stepsize parameter. Note that Equation (7) has no guarantee of the non-negativity of $\mathrm{H}$. Hence in each iteration all negative values in $\mathrm{H}$ are set to zero. Then at the end of each iteration, each column $h_{j}$ is normalized to have unity maximum.

To sum up, $\mathrm{S}^{i}$ and $\mathrm{H}$ are optimized alternatively by using the multiplicative update rule and the projected gradient descent respectively, as described in Algorithm 1. $\mathrm{H}$ is initialized as a random non-negative matrix and $\mathrm{S}^{i}$ is initialized as a random diagonal non-negative matrix,335 which is a widely-used initialization strategy for NMF-based methods (Kuang et al. 2012). Of note, for $\mathrm{S}^{i}$, its iteration under Equation (6) is guaranteed to converge to its global minimum quickly. However, for $\mathrm{H}$, no global minimum can be guaranteed due to the non-convex constraint on $h_{j, 30}$ even though the step size $\mu$ is small enough. Besides, the randomness of initialization leads to changes in results of each computation. Thus multiple computations are needed to select the best result.

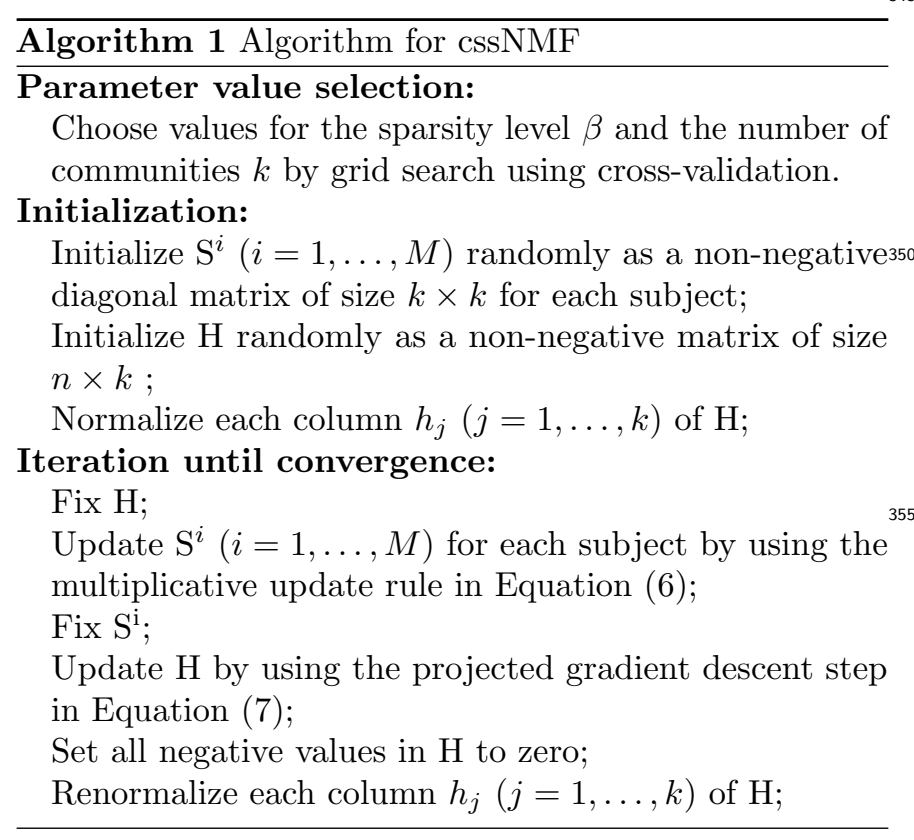

\subsection{Datasets}

\subsubsection{Simulated fMRI dataset}

The simulated fMRI dataset with ground-truth used in our experiment is from Eavani et al. (2015), which was generated at a repetition time (TR) value of 3 seconds by using the Netsim software (Smith et al., 2011) based on dynamic causal modeling (DCM) (Friston et al. 2003). Here, we use the simulated fMRI time series with 120 time-points of the first 45 nodes ${ }^{1}$ The overall community structure of all subjects consists of eight communities with the size varying from 3 nodes to 10 nodes. And these communities share several overlapping nodes to different extents. Besides, the inter-subject variability in community strengths was introduced by varying the activation strengths of communities for each individual. Please refer to the work of Eavani et al. (2015) for a detailed description of this dataset about its generation and its underlying network configuration.

\subsubsection{CoRR dataset}

To evaluate the proposed method, in comparison with the other four methods, in terms of reproducibility and reliability, the Hangzhou Normal University (HNU) dataset containing repeated measurements of rs-fMRI data from the Consortium for Reliability and Reproducibility (CoRR) (Zuo et al. 2014) is applied here. Details about scan parameters and other information of this dataset can be found in http://dx.doi.org/10.15387/fcp_indi.corr.hnu1. Briefly, thirty healthy participants (15 females) were recruited with the mean age of 24 years old ( $\mathrm{SD}=2.41)$. Each session of the rs-fMRI data was acquired by following the same procedure. Participants were instructed to open eyes with a fixation and the acquisition lasts for 10 mins with a TR value of 2 seconds, thus resulting in 300 time-points. The first two sessions are used in our experiment, which are three days apart. Each participant provided written informed consent, and ethical approval of data collection was obtained from the ethics committee of the Center for Cognition and Brain Disorders (CCBD) at Hangzhou Normal University.

\subsubsection{Beijing_Zang dataset}

Another real rs-fMRI dataset of twenty subjects, Beijing_Zang, is also used in order to assess the consistency of

\footnotetext{
${ }^{1}$ The original dataset consists of 50 nodes including a cluster of last 5 nodes that are negatively connected with the other nodes. Since the negative association is not our concern, we exclude the last 5 nodes in our experiment.
} 
results across distinct datasets. It is part of the 1000 Functional Connectomes Project (Biswal et al. 2010), which can be downloaded from http://fcon_1000.projects.nitrc org. During the acquisition of 7.5 mins, participants performed no task in particular with their eyes closed.

\subsection{Data preprocessing}

The real rs-fMRI datasets were preprocessed by using the pipelines provided by the Data Processing Assistant for Resting-State fMRI (DPARSF) (Yan and Zang, 2010). The CoRR data was preprocessed following the steps used in Chen et al. (2015). Specifically, the first 5 echoplanar imaging (EPI) volumes were discarded and then the steps of slice timing and realignment were conducted. To further reduce the effect of head motion and physiological artifacts, we performed regressions by using the Friston-24 parameter model together with cerebrospinal fluid (CSF) and white matter (WM) signals as the covariates. Then ${ }^{405}$ we regressed out linear and quadratic trends for individual rs-fMRI data. Afterwards, the resulting data were spatially normalized to the Montreal Neurological Institute (MNI) space by DARTEL procedure, due to the availability of corresponding T1 images of the participants, and then resampled to a voxel size of $3 \times 3 \times 3 \mathrm{~mm}$. Then the preprocessed data were spatially smoothed with a $4 \mathrm{~mm}$ full width half maximum (FWHM) Gaussian kernel and bandpass filtered $(0.01-0.1 \mathrm{~Hz})$. For both sessions, all subjects meet the criteria that head motion is less than $3 \mathrm{~mm}$ of translation and 3 degree of rotation. The Beijing_Zang dataset was preprocessed similarly, as described in Li and Wang (2015). Finally, the automated anatomical labeling (AAL) template (Tzourio-Mazoyer et al., 2002) defining 90 ROIs on the brain cortex was applied and the mean fMRI time series of each ROI was extracted for each individual of both datasets.

\subsection{Parameter selection}

For both simulated fMRI and real rs-fMRI data, $\operatorname{cssNMF}_{425}$ detects the group-level overlapping community structure based on individual association matrices derived by NASR The parameter $\lambda$ in NASR is predefined to 0.1 empirically for all datasets (Li et al. 2016a b). The two parameters in the cssNMF model (i.e., community number $k$ and sparsity 430 level $\beta$ ) are determined by a grid search with a two-fold cross-validation procedure. The test error used in the cross-validation process is defined as follows:

$$
\text { Test error }=\frac{\sum_{i=1}^{N_{\text {test }}}\left\|\mathrm{G}_{\text {test }}^{i}-\mathrm{H}_{\text {train }} \mathrm{S}_{\text {test }}^{i} \mathrm{H}_{\text {train }}^{\mathrm{T}}\right\|_{\mathrm{F}}^{2}}{\sum_{i=1}^{N_{\text {test }}}\left\|\mathrm{G}_{\text {test }}^{i}-\overline{\mathrm{G}}_{\text {test }}\right\|_{\mathrm{F}}^{2}} \text {. }
$$

The whole dataset is divided into a training set and a testing set, and $N_{\text {test }}$ is the size of the testing set. $\mathrm{G}_{\text {test }}^{i}$ and $\mathrm{S}_{\text {test }}^{i}$ denote the association matrix and its corresponding weight matrix of the $i$ th subject, while $\overline{\mathrm{G}}_{\text {test }}$ is the mean association matrix across all subjects in the testing set. Equation (8) measures the reconstruction error of the testing set by using the membership matrix $\mathrm{H}_{\text {train }}$ learned on the training set, divided by its variation. By using this measure, we aim to find the appropriate parameter values at which the increment of information carried by $\mathrm{H}$ and $\mathrm{S}^{i}$ only results in negligible gain in generalizability. In other words, the parameters are determined when the increase of $k$ or the decrease of $\beta$ causes little drop in the test error.

As described before, multiple runs are needed for cssNMF due to the random initializations and the non-convex constraint. Therefore, for each computation in the experiment, we run the cssNMF algorithm ten times and select the best run with the minimum value of the objective function in Equation (4) for the subsequent analysis.

\subsection{Competing methods}

Infomap and modularity optimization are two leading methods aiming to find an optimal non-overlapping partition of a network based on different fitness functions. Infomap seeks a minimization of the description length defined by a map equation (Rosvall and Bergstrom, 2008), while modularity optimization maximizes the modularity of a network partition (Newman, 2006). Different from cssNMF, Infomap and modularity optimization identify the group-level community structure based on the groupaveraged association matrix, instead of all individual association matrices, and result in a non-overlapping community structure. In this experiment, the edge density of the input association matrix for these two methods was adjusted by thresholding the association matrix so as to vary the number of communities of the partition. The Infomap algorithm was implemented using the code available on http://www.tp.umu.se/ rosvall/code.html. The modularity optimization was performed by using the Louvain method implemented in the Brain Connectivity Tool- 
box (Rubinov and Sporns, 2010). For both methods, we launched 1000 runs and selected the best results with minmum description length for Infomap and maximum modularity for modularity optimization.

By contrast, sICA and tICA take the fMRI time series directly as input and identify sub-networks with overlap. For this study, the fMRI time series of all subjects are concatenated and the dimensionality is reduced by using PCA (Smith et al. 2012). After that, these fMRI data are fed into the fastICA algorithm (Hyvärinen 1999) implemented by the FastICA software available from http://www.cis.hut.fi/projects/ica/fastica/ with

varying number of components for both sICA and tICA.

Besides, to further test the performance of the proposed 480 cssNMF approach, in this experiment all network-based algorithms, i.e., cssNMF, Infomap and modularity optimization are also carried out based on the association matrix constructed by using the Pearson correlation. Specifically, for each pair of ROIs, the functional connectivity is calculated by using Pearson correlation and the correlations are then transformed to Fisher's z values. All negative values are flipped and all diagonal entries are set to zeros, a $^{\text {as }}$ usually done in practice (Power et al. 2011, Eavani et al. 2015). The whole framework to carry out cssNMF, Infomap, modularity optimization, sICA and tCA is shown ${ }^{490}$ in Figure 1

\subsection{Evaluation metrics}

For a direct comparison, the output of all these methods are converted into a community membership matrix $\mathrm{H}$. To be specific, each community/component forms a column of the membership matrix and the label assignments of all nodes produced by Infomap and modularity optimization are converted into a binary version of the membership matrix. Before quantitatively comparing any two sets of communities, a graph matching procedure is conducted by using the Hungarian algorithm (Lovász and Plummer 1986), since the order of the resulting communities may be different across computations and methods.

For any two resulting membership matrices $\mathrm{H}$, their similarity is calculated firstly for each pair of matched communities by using the normalized inner product, and $_{500}$ then averaged over all communities. Particularly, for the simulated data, the performance of all methods is usually evaluated by measuring the similarity between the experimental results and the ground-truth of the underlying group-level community structure to depict the degree of their correspondence.

Furthermore, for the simulated data, since the groundtruth is binary and gives a clear membership of each community while the numerical results of cssNMF, sICA and tICA are continuous and only indicate the degree of participation in a community of each node, we apply a threshold $\tau$ to transform the results into binary variables to calculate the accuracy. For each community, the accuracy is calculated by

$$
\operatorname{accuarcy}\left(h_{j}\right)=\frac{T P+T N}{T P+F P+T N+F N} .
$$

where TP, TN, FP, FN denote true positive, true negative, false positive and false negative respectively. It measures the ratio of the number of nodes that are correctly identified $(\mathrm{TP}+\mathrm{TN})$ to the total amount of nodes $(\mathrm{TP}+\mathrm{FP}+\mathrm{TN}+\mathrm{FN})$. The overall accuracy across all communities is then computed by accuracy $(\mathrm{H})=\left(\sum_{j=1}^{k} \operatorname{accuracy}\left(h_{j}\right)\right) / k$.

Similarly, the analysis of the inter-subject variability obtained by cssNMF is also conducted in terms of similarity and accuracy. For each community, the similarity to the corresponding ground-truth measured by the normalized inner product indicates the ability of cssNMF to capture the individual differences in community strength. Then the overall accuracy across all communities with regard to the binary ground-truth is further computed by Equation (9).

For the resulting group-level community structure on the real rs-fMRI data, a higher sparsity often indicates a structure that is easier to understand. The sparsity of a community $h_{j}$ is computed as follows (Hoyer, 2004):

$$
\operatorname{sparsity}\left(h_{j}\right)=\frac{\sqrt{n}-\left\|h_{j}\right\|_{1} /\left\|h_{j}\right\|_{2}}{\sqrt{n}-1},
$$

where $n$ denotes the dimension of $h_{j}$. It reaches the maximum value of one when there is only one non-zero element and the minimum value of zero when all elements are of the same value. The overall sparsity of the whole community structure $\mathrm{H}$ is then obtained by averaging the sparsity across all communities, i.e., $\operatorname{sparsity}(\mathrm{H})=$ $\left(\sum_{j=1}^{k}\right.$ sparsity $\left.\left(h_{j}\right)\right) / k$.

Another important aspect of the evaluation of the grouplevel community structure is its consistency across time, 
(A)

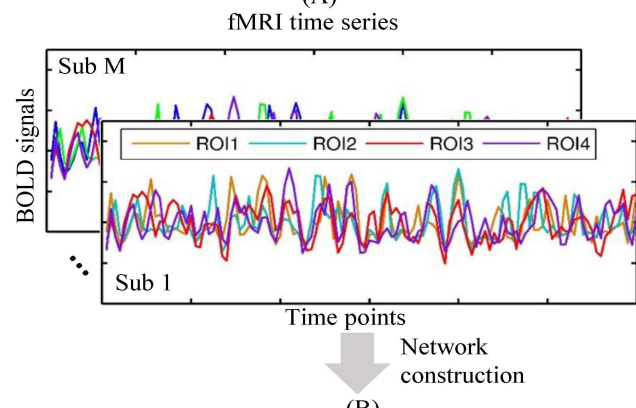

(B)
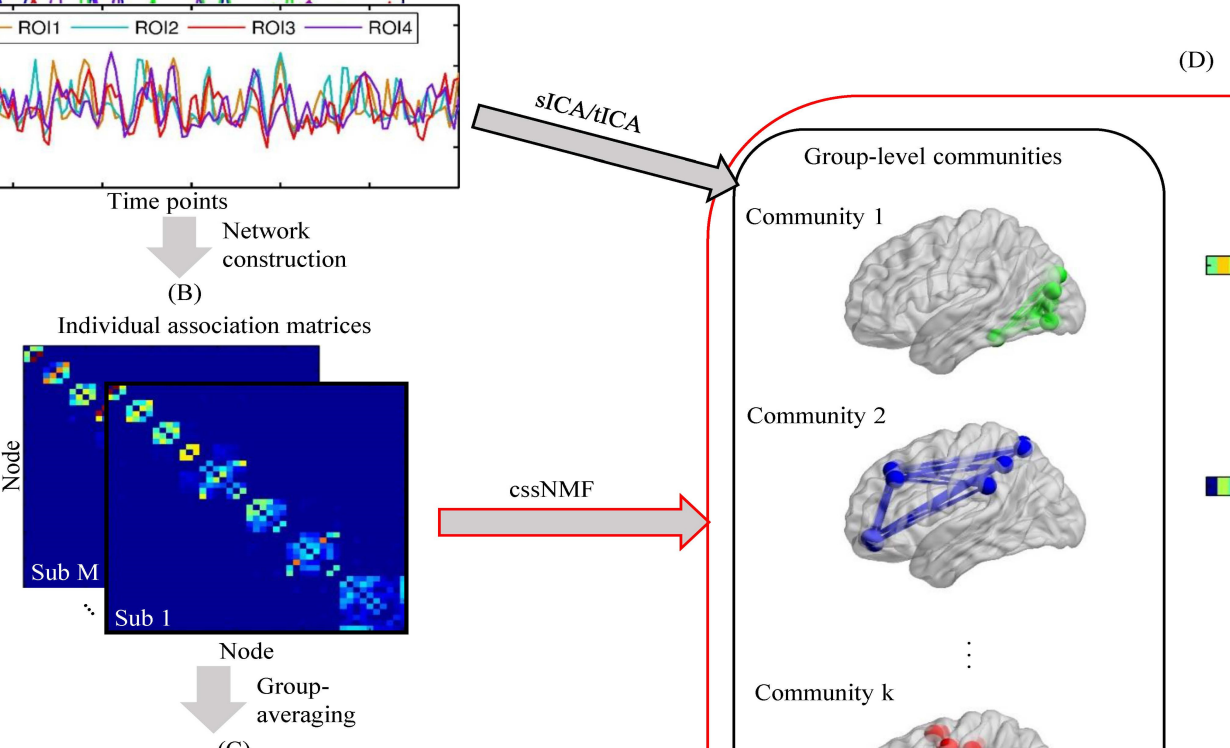

(D)
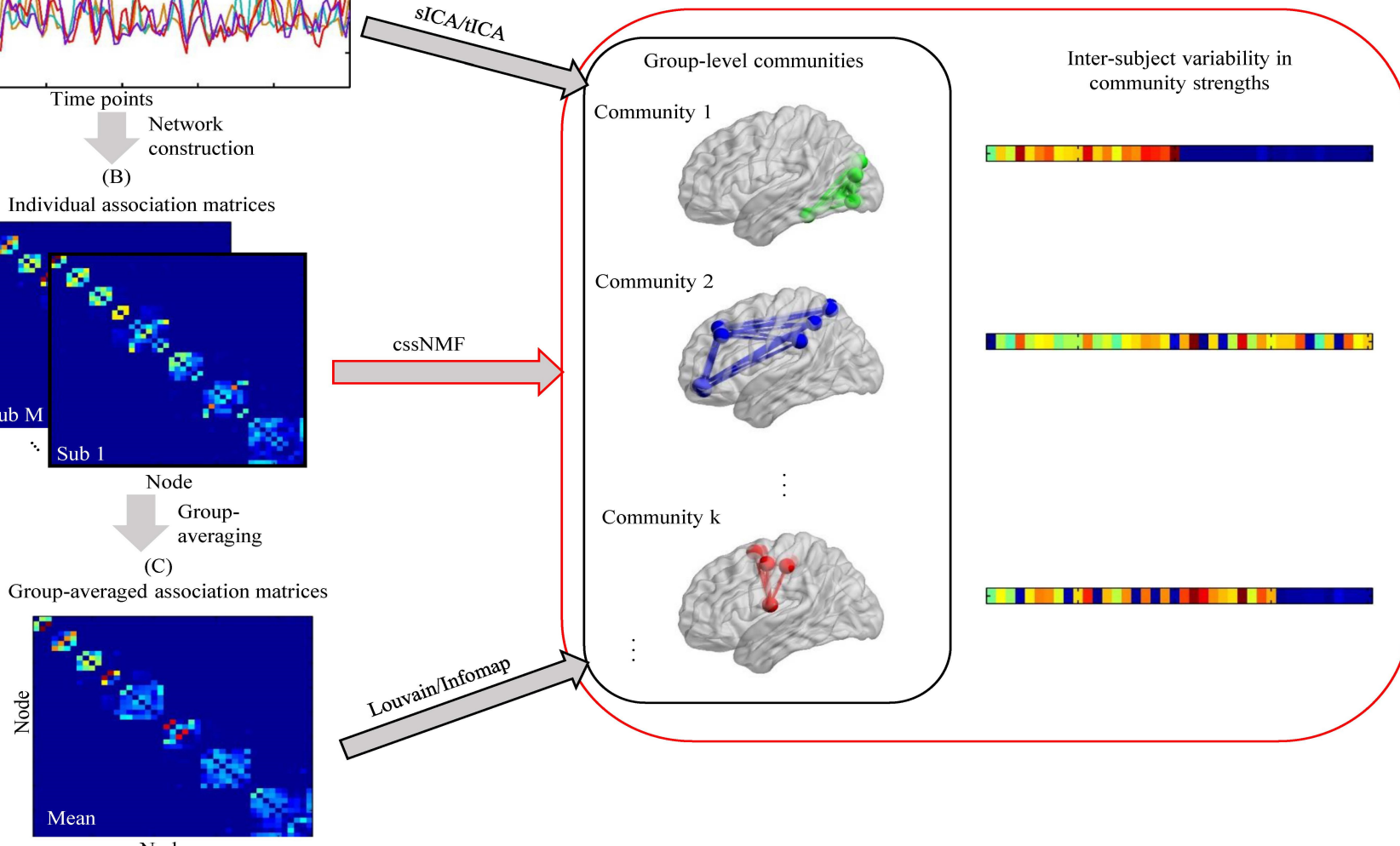

Figure 1: Schematic illustration of the experiment framework of cssNMF, Infomap, Louvain, sICA and tICA. (A) The fMRI time series of each ROI are extracted for all subjects. (B) Based on the fMRI time series, the whole-brain network is constructed for each subject by calculating its association matrix. (C) The group-averaged association matrix across all subjects is calculated. (D) sICA/tICA and Infomap/Louvain identify the group-level networks/communities in the black box based on the concatenated fMRI time series and on the group-averaged association matrix respectively, while cssNMF factorizes all these individual association matrices collectively into group-level communities as well as inter-subject variability in community strengths in the red box.

subjects or datasets, i.e., reproducibility. For two sets of matched communities, reproducibility is defined as the mean similarity across all communities measured by normalized inner product. Firstly, cross-session reproducibility is computed based on results of the two sessions from the CoRR dataset of the same group of subjects. It tests whether the obtained community structure is stable across time. Secondly, for cross-subject reproducibility, we split the data of the first session from the CoRR dataset into two halves of separate subjects (each half containing 15 subjects) and calculate the consistency between the two halves. This procedure is repeated 100 times and the mean across these computations is taken as the final measure of cross-subject reproducibility. Finally, the Beijing_Zang dataset is used to compute cross-data reproducibility. In contrast, a desirable property of the individual differences derived by cssNMF is not only the ability to retain stable across time but also being sensitive to the differences between subjects, which is characterized by test-retest reliability (Zuo et al., 2010). In other words, a high test-retest reliability means a low intra-subject variability but a high inter-subject variability. Test-retest reliability has drawn great attention in fMRI studies recently (Shehzad et al. 2009 Cao et al. 2014; Zuo et al., 2014) and is often computed by using the intra-class correlation coefficient (ICC) index (Shrout and Fleiss, 1979):

$$
I C C(C, 1)=\frac{M S_{B}-M S_{E}}{M S_{B}+(m-1) * M S_{E}},
$$

where $m=2$ in our experiment denotes the number of 
(A)

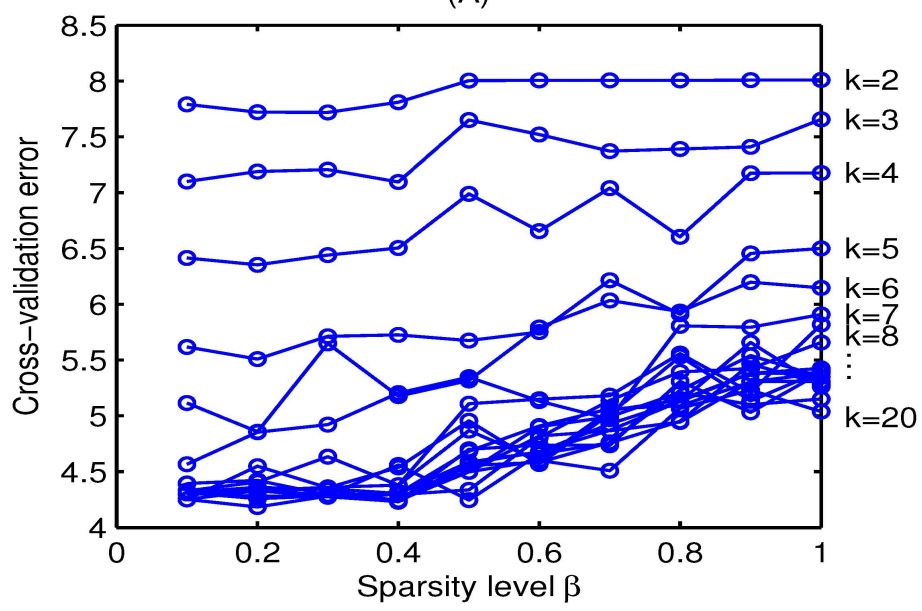

(B)

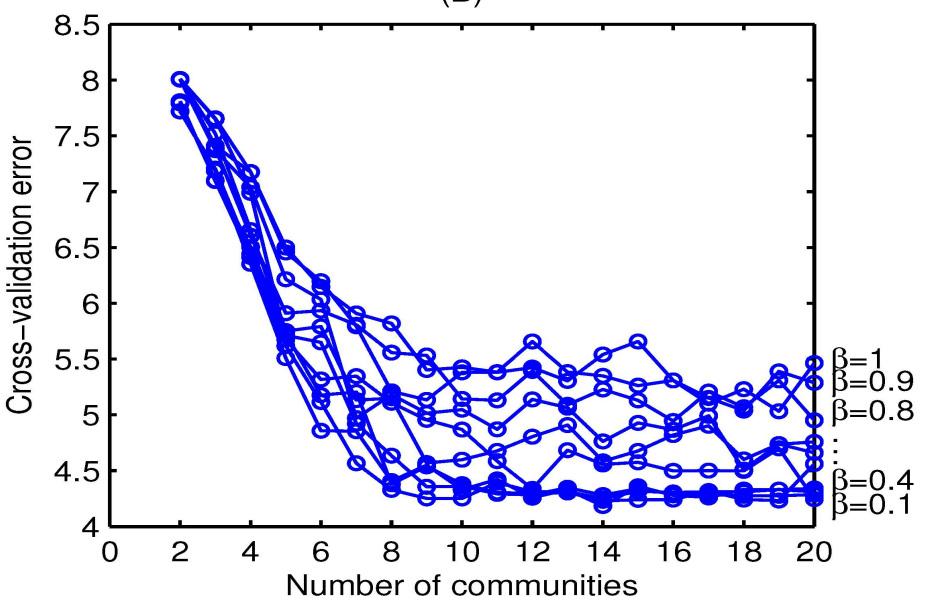

Figure 2: Parameter selection for the cssNMF method on the simulated data by grid search using cross-validation based on NASR-based association matrices. (A) Cross-validation error (Equation (8) vs. sparsity level $\beta$. (B) Cross-validation error vs. number of communities $k$.

repeated sessions. $M S_{B}$ is the mean square (i.e., variance) between subjects in community strengths and $M S_{E}$ represents the mean squared error (please refer to Zuo et al. (2010) for detailed computation and interpretation of $M S_{B}{ }^{505}$ and $M S_{E}$ ). Negative ICC values are set to zero as usually done in practice (Braun et al. 2012). The ICC values are equally divided into five levels, which are $(0,0.2]$ (slight), $(0.2,0.4]$ (fair), $(0.4,0.6]$ (moderate), $(0.6,0.8]$ (substantial) and $(0.8,1]$ (almost perfect) separately from low to high ${ }^{510}$ (Landis and Koch, 1977). Results of the inter-subject variability in community strengths on CoRR dataset are used and the strengths of each community are collected from all $M$ subjects, i.e., $s_{j}=\left[s_{j}^{1}, s_{j}^{2}, \ldots, s_{j}^{i}, \ldots, s_{j}^{M}\right], j=1, \ldots, k$ for each session and each element $s_{j}^{i}$ denotes the strength of ${ }^{515}$ community $j$ of the $i$ th subject. The ICC index is computed for each pair of $s_{j}$ and then averaged across all communities. Before that, we use a simple rescaling technique to standarize all elements in each $s_{j}$ so as to have the same range between zero and one. Because for each subject it is ${ }^{520}$ the relative weight of the strength rather than its absolute value in one community that we are concerned with, $s_{j}^{i}$ is rescaled as follows:

$$
\left(s_{j}^{i}\right)^{\prime}=\frac{s_{j}^{i}-\min \left(s_{j}\right)}{\max \left(s_{j}\right)-\min \left(s_{j}\right)},
$$

where $\left(s_{j}^{i}\right)^{\prime}$ is the rescaled value of the original $s_{j}^{i}$.

\section{Results}

\subsection{Results on simulated fMRI data}

To determine the values of parameters in the cssNMF method, a grid search was conducted by using a two-fold cross-validation. Results based on NASR-derived association matrices are shown in Figure 2. The number of communities $k$ varies from 2 to 20 with a stepsize of 1 and the sparsity level $\beta$ varies from 0.1 to 1 with a stepsize of 0.1 . The selection of $k$ seems straightforward since the test error decreases significantly until $k$ reaches 8 , which is in accordance with the ground-truth of the simulated data. Besides, the test error stays relatively stable as $\beta$ decreases from 0.4 to 0.1 for most values of $k$. Thus, $k$ and $\beta$ were set to 8 and 0.4 respectively for the simulated data. The same process was done on association matrices derived by the Pearson correlation and the results are shown in Figure S1. Similarly, for most cases the test error fluctuates frequently until $\beta$ decreases to 0.55 , where the test error stays quite stable as $\beta$ continues decreasing. Thus $\beta=0.55$ was selected in this experiment. For the selection of $k$, the test error drops sharply until $k$ increases to 6 . However, for a plain comparison between NASR-derived results and correlation-based results, $k$ was set to 8 , i.e., the same as the value selected for the NASR-derived ones.

For network-based community detection algorithms, cssNMF, Infomap and modularity optimization, an accurate brain network construction is a crucial pre-step. Figure 3 illustrates the mean association matrix averaged over all 


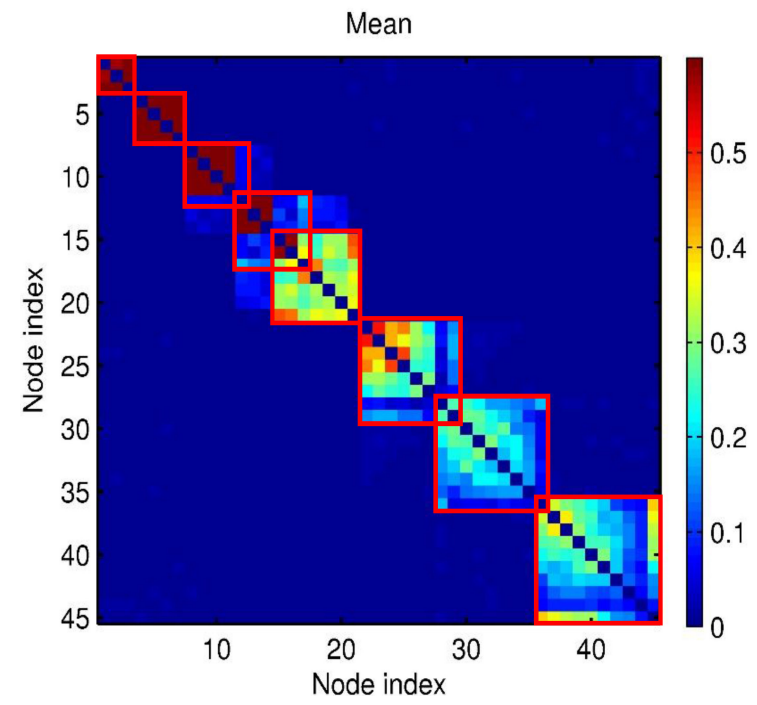

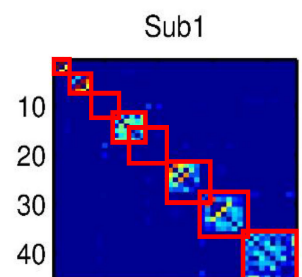

$1020 \quad 30 \quad 40$

Sub3

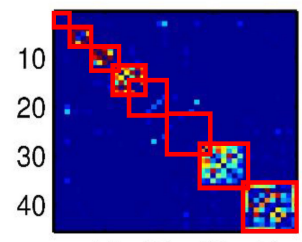

$10 \quad 20 \quad 30 \quad 40$

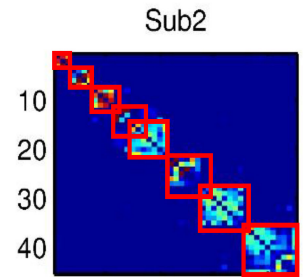

$102030 \quad 40$

Sub4

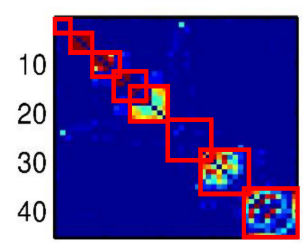

$10 \quad 2030 \quad 40$

Figure 3: Association matrices derived by NASR on the simulated dataset. The mean association matrix as well as the individual association matrices of four randomly selected subjects are shown. The red contours indicate the overall ground-truth network configuration of the simulated dataset.

subjects and the individual association matrices of four randomly selected subjects constructed by the NASR method 560 The corresponding association matrices derived by the Pearson correlation are shown in Figure S2. The red contours indicate the overall ground-truth network configuration of the simulated dataset. It can be seen that by calculating the non-negative association between nodes, NASR is565 able to depict the underlying network configuration with individual differences for each subject nicely and derives non-negative sparse association matrices. However, the association matrices produced by the Pearson correlation are quite dense where almost all connection weights are 570 non-zero. Besides, spurious negative values often appear in individual association matrices, which are hard to interpret for the simulated dataset where networks are not negatively related.

Based on the individual association matrices, the overall community structure, as well as the inter-subject variability in community strengths, is obtained by cssNMF. For ICA methods, Infomap and modularity optimization, only the group-level community structure is derived based either on concatenated fMRI time series of all subjects or on the group-averaged association matrix. The group-level community structures from the ground-truth and identified by these methods are shown in Figure 4(A), where each column represents a community membership vector. Here, the number of communities $k$ for ICA methods were set to 8 according to the ground-truth, while for Infomap and modularity optimization, different values of $k$ were obtained with varying edge-densities of the association matrix and the results with the highest similarity to the ground-truth were shown. The first row in Figure 4(A) shows the overall community structure from the ground-truth and those derived by sICA and IICA. The second row and the third row display the overall community structures identified by cssNMF, Infomap and modularity optimization, based on the NASR-derived association matrices and on the correlation-based association matrices respectively. Visually, the overall communities derived by cssNMF show the highest resemblance to the ground-truth on both NASRderived and correlation-based results compared to the other methods, since cssNMF uniquely learns a sparse overlapping community structure without negative values. By contrast, although ICA methods, especially tICA, are also able to discover the overlapping community structure, they tend to produce a much denser result with some spuriously high or negative values of community membership, whereas Infomap and modularity optimization fail to identify the overlapping community structure. The similarities between the resulting community structures of all methods and the ground-truth community structure are quantitatively measured by using normalized inner-product separately, as shown in Figure 4(B). Note that we also launched the cssNMF method with $\beta=0$, where no extra sparsity is enforced, to observe how its performance is affected by the sparsity constraint. It reveals that the network-based meth- 

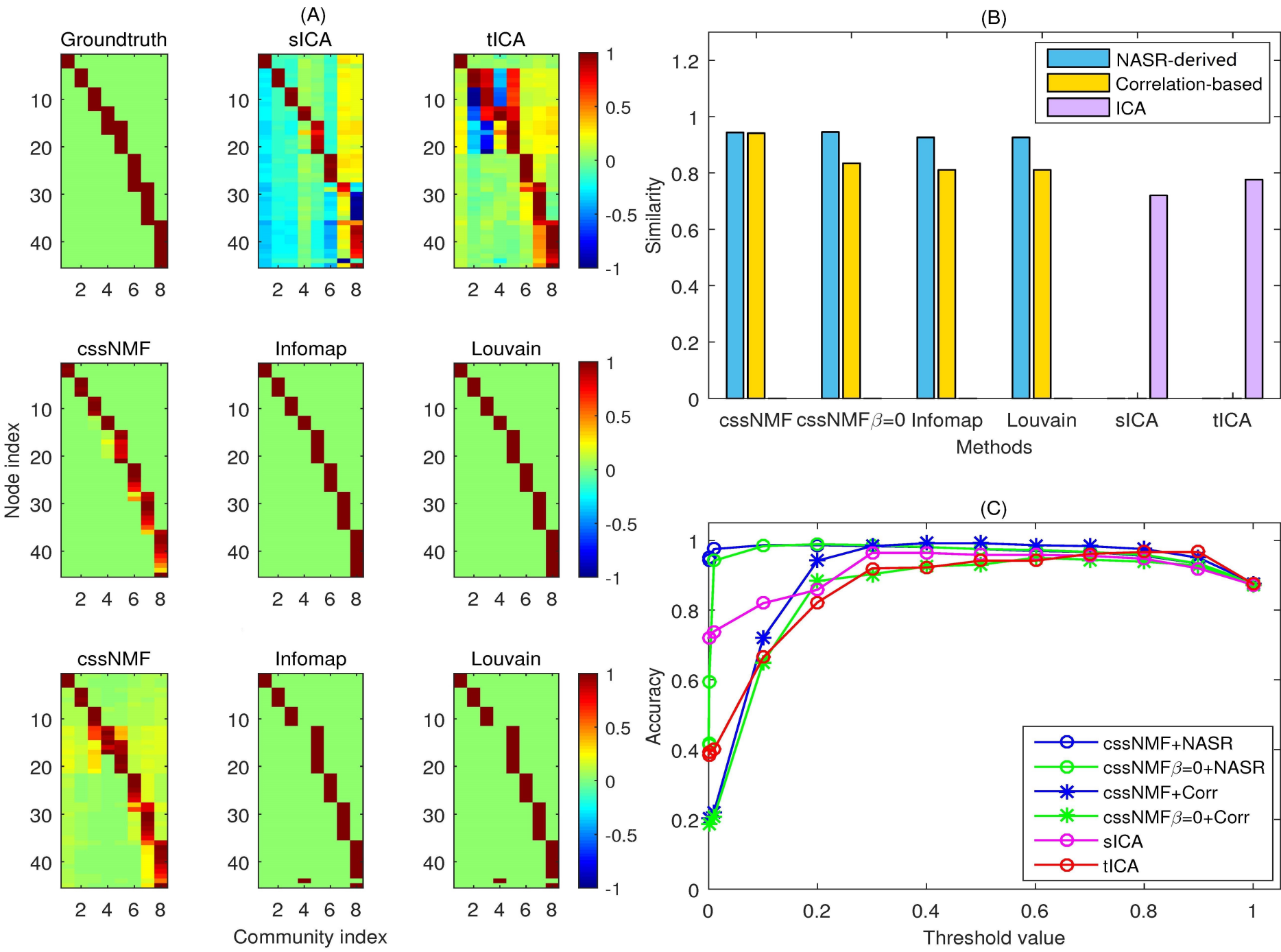

Figure 4: Results of the group-level community structure on the simulated dataset. (A) Illustration of the group-level communities/networks. The top row displays the ground-truth and those identified by sICA and tICA. The middle and bottom rows display the communities identified by cssNMF, Infomap and Louvain method based on the NASR-derived and correlation-based association matrices respectively. (B) Similarity between the ground-truth communities and those identified by cssNMF, Infomap, Louvain method, sICA, and tICA separately. For cssNMF, cssNMF without sparsity ( $\operatorname{cssNMF} \beta=0$ ), Infomap and Louvain method, results on both the NASR-derived association matrices and on the correlation-based ones are shown here. (C) Accuracy of the continuous methods including cssNMF (under different conditions), sICA and tICA in detecting the community structure with varying thresholds.

ods, i.e., cssNMF, Infomap and modularity optimization perform better than tICA (0.776) and sICA (0.720) in terms of similarity, regardless of which method is used to con-600 struct the association matrix. Among these network-based methods, cssNMF achieves the highest score in general. Specifically, based on the NASR-derived association matrices, Infomap and modularity optimization reach a similarity of 0.926 , while cssNMF reaches a similarity of 0.944 (with605 sparsity) and 0.945 (without sparsity). The advantage of cssNMF is more evident than the other methods when performed on correlation-based association matrices, with a similarity of 0.941 (with sparsity) and 0.835 (without sparsity), followed by Infomap and modularity optimization both with a similarity of 0.811 . Finally, for matrix factorization-based methods which result in a continuous membership matrix, including cssNMF, sICA and tICA, the accuracy of the identified community structure compared to the ground-truth is measured by using Equation (9) against a wide range of thresholds $\tau$ (varying from 0.0001 to 1 ). It shows that the NASR-based community structure identified by cssNMF with sparsity is significantly less sensitive to the selection of threshold than the other 
(A)
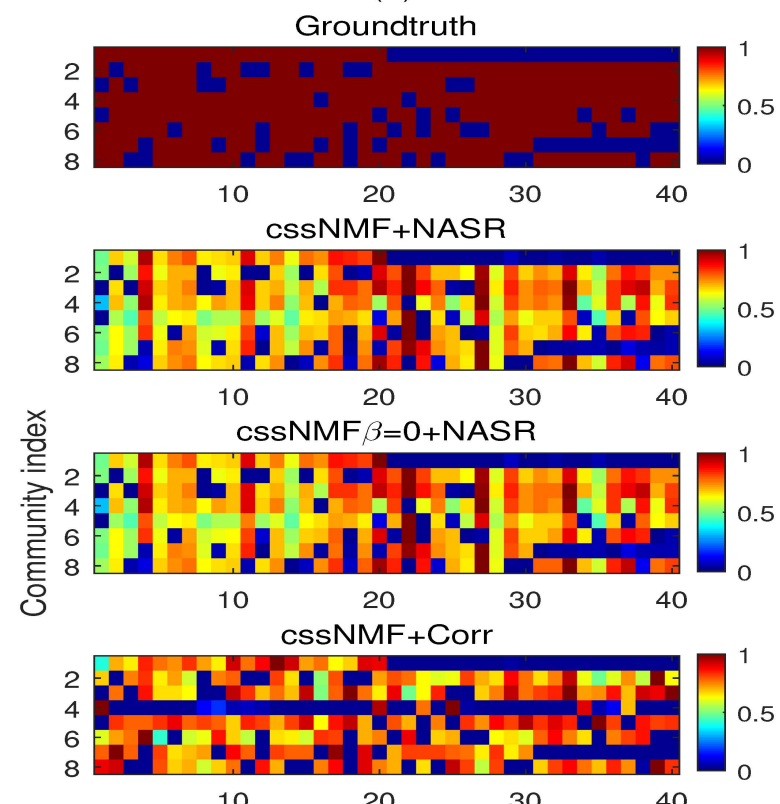

$\operatorname{cssNMF} \beta=0+$ Corr

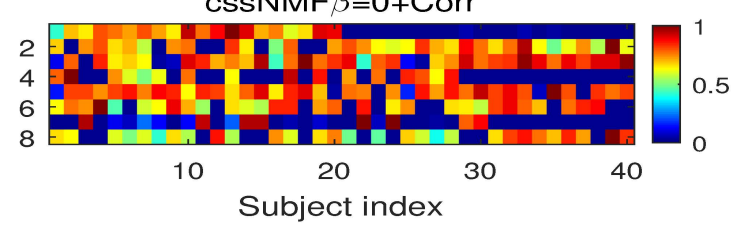

(B)

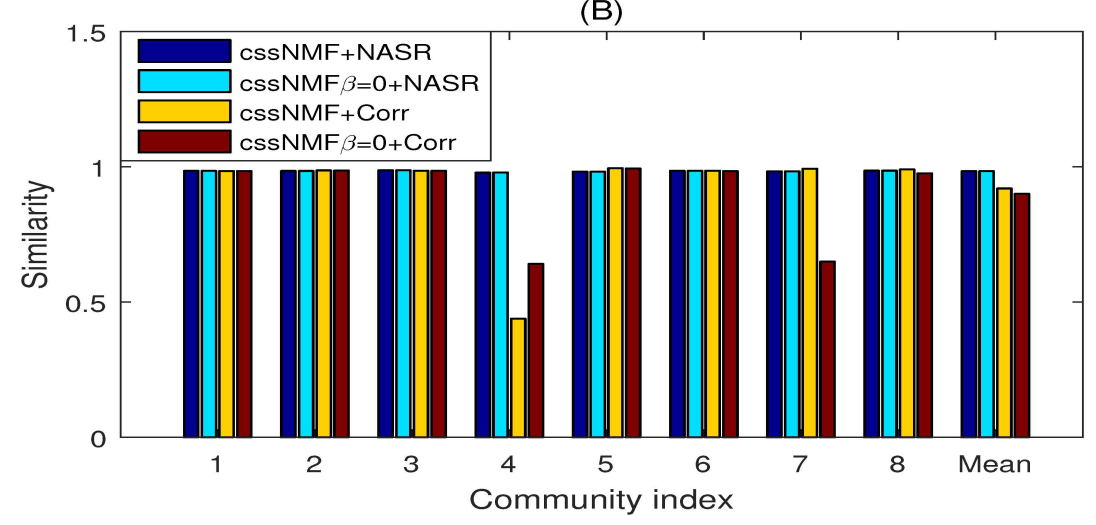

(C)

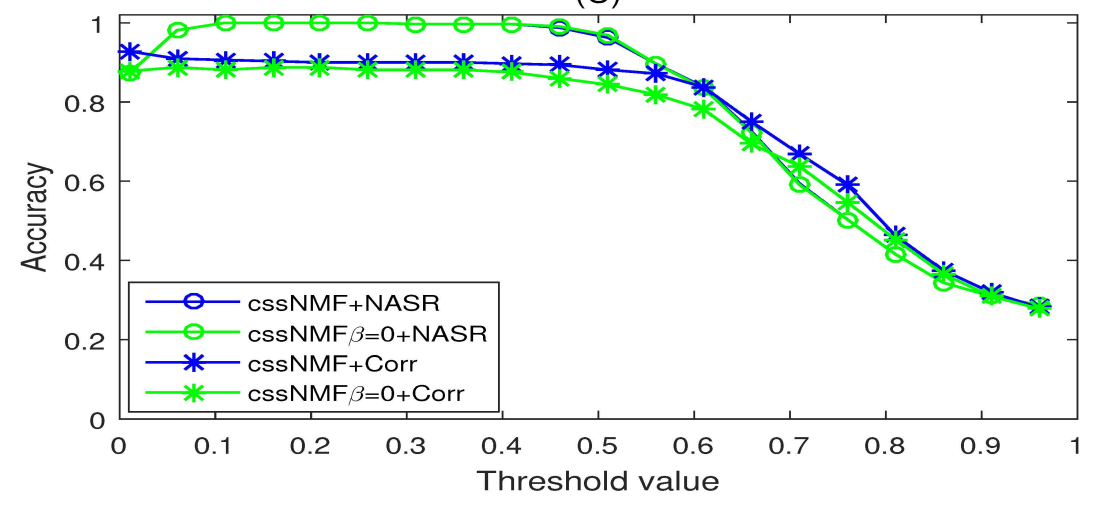

Figure 5: Results of the inter-subject variability on the simulated dataset captured by cssNMF under different conditions. (A) Illustrations of the binary ground-truth and the inter-subject variability in community strengths detected by cssNMF and cssNMF without sparsity ( $\operatorname{css} N M F \beta=0$ ), where NASR and Corr denote the results are obtained on NASR-derived and on correlation-based association matrices respectively. (B) Similarity between the detected inter-subject variability and the ground-truth of individual communities separately with the mean similarity across all communities. (C) Accuracy in detecting the inter-subject variability with varying thresholds.

methods, since it stays stable above 0.9 even when $\tau$ is close to zero. However, the accuracy of cssNMF without sparsity drops to 0.4 when $\tau$ is below 0.01 , although it performs slightly better than cssNMF with sparsity in terms of 630 similarity. By contrast, the accuracy of the other methods rises rapidly until $\tau$ reaches above 0.2 . It suggests that both NASR and cssNMF with sparsity contribute to the robustness of the identified community structure and lead to easier interpretation of the results.

Different from the other methods, in addition to the group-level community structure, cssNMF is also able to capture the inter-subject variability in terms of community strength. These results as well as the corresponding binary ${ }_{640}$ ground-truth are illustrated in Figure 5(A), where each row represents the variations in community strengths of all subjects for one community. Though varied in values of the community strengths, the experimental results of cssNMF under different conditions all show similar patterns to the binary ground-truth. Quantitative measures in terms of similarity and accuracy are shown in Figures $\mathbf{5}(\mathbf{B})$ and (C) respectively. Overall, cssNMF under different conditions consistently achieves a surprisingly high similarity of above 0.9 , although it performs slightly better on association matrices derived by NASR (0.984) than on those derived by the Pearson correlation (0.920 with sparsity and 0.900 without sparsity). In general, the sparsity level $35 \beta$ has little impact on the results in terms of the intersubject variability for most communities. Similarly, the accuracy of the NASR-based results is higher than that of the correlation-based results and it reaches up to almost 1 in a wide range of $\tau$ (around 0.1 to 0.5 ). It means that for all subjects cssNMF can correctly decide whether a community is recruited in its whole-brain network, thus indicating that cssNMF is capable of effectively reflecting the individual differences in the degree of participation (community strength) of a community. 
(A)

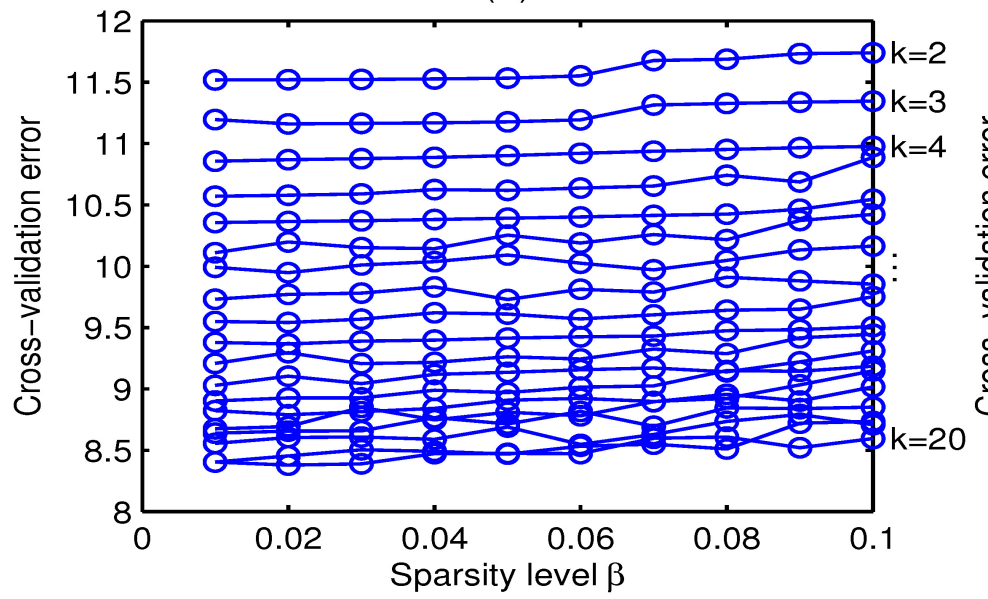

(B)

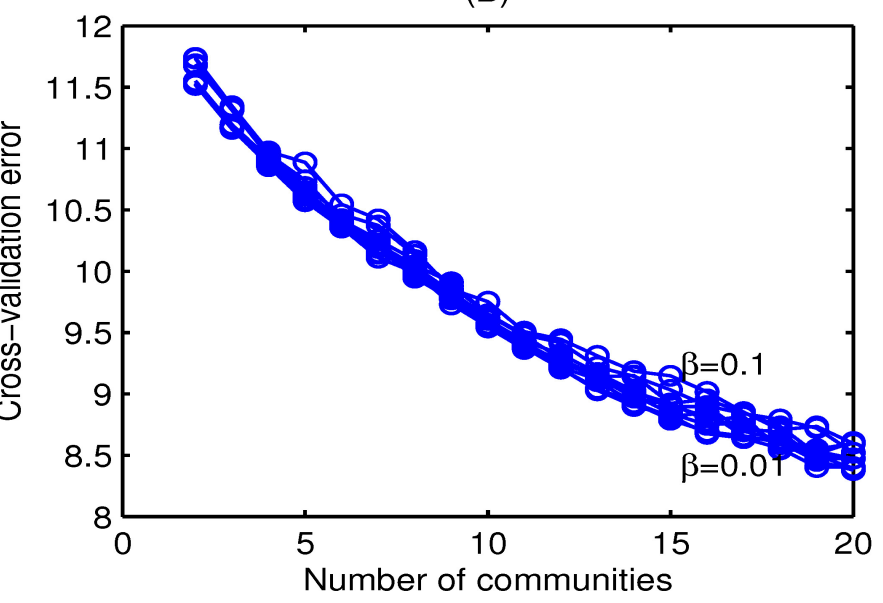

Figure 6: Parameter selection for the cssNMF method on the real rs-fMRI dataset by grid search using cross-validation. (A) Cross-validation error (Equation (8)) vs. sparsity level $\beta$. (B) Cross-validation error vs. number of communities $k$.

To sum up, the proposed cssNMF method outperforms the other competing methods on both NASR-derived and correlation based association matrices in terms of iden- 675 tifying the group-level community structure, as well as extracting the inter-subject variability via the community strength. Since how the association matrix is constructed is not the focus of this study and all network-based community detection methods perform better on NASR-derived association matrices in the experiment on the simulated data, in the following experiment on the real fMRI data all network-based methods are only applied to the NASRderived association matrices.

\subsection{Results on real rs-fMRI data}

For the real rs-fMRI experiment, the parameter value selection for cssNMF was performed on the first session of the CoRR data. The corresponding results are shown in Figure 6, where the number of communities $k$ ranges from690 2 to 20 with a stepsize of 1 and the value of $\beta$ was narrowed down to a range of 0.01 to 0.1 with a stepsize of 0.01 , since the test error declines significantly as $\beta$ decreases from 1 to 0.1 . As can be seen, the test error only slightly changes as $\beta$ decreases from 0.07 to 0.01 for most conditions695 of $k$. However, unlike the simulated data, the selection of $k$ seems unclear since the test error keeps dropping significantly as $k$ increases. Thus we applied $\beta=0.07$ and $k$ varying from 2 to 20 to all real fMRI datasets. For Infomap and modularity optimization, we were able to700 obtain results with $k$ varying from 17 to 28 and from 7 to
24 (not uniformly spaced) respectively, as we decreased the edge density of the association matrices from 1 to 0.02 . For sICA and tICA, $k$ was varied from 2 to 20 with a stepsize of 1 .

The group-level communities derived by all these methods on the first session of CoRR real data with a moderate size of $k=9$ and a larger size $k=18$ (where we were able to obtain the results of all methods) are shown in Figures 7(A) and (B) respectively. (Note that for Infomap we were only able to obtain the group-level communities with $k \geq 17$ on this dataset, so the 9 communities derived by Infomap shown in this paper are the ones obtained with Infomap and modularity optimization produce sparse but totally non-overlapping community structures, while the ones derived by sICA and IICA are overlapping but rather dense. By contrast, cssNMF appears to seek a trade-off between these two types of methods by identifying a sparse but overlapping community structure. To depict such differences more accurately, we quantified the sparsity of the overall community structures by using Equation (10) for all methods based on the large repeated split-half data (containing 200 datasets). For each value of $k$, the sparsity measure is calculated for each dataset and then averaged across all datasets. Results are shown in Figure 8. Not surprisingly, Infomap and modularity optimization achieve the highest sparsity in the group-level community structure with varying values of $k$, followed closely by cssNMF, while sICA and tICA fall far behind. 

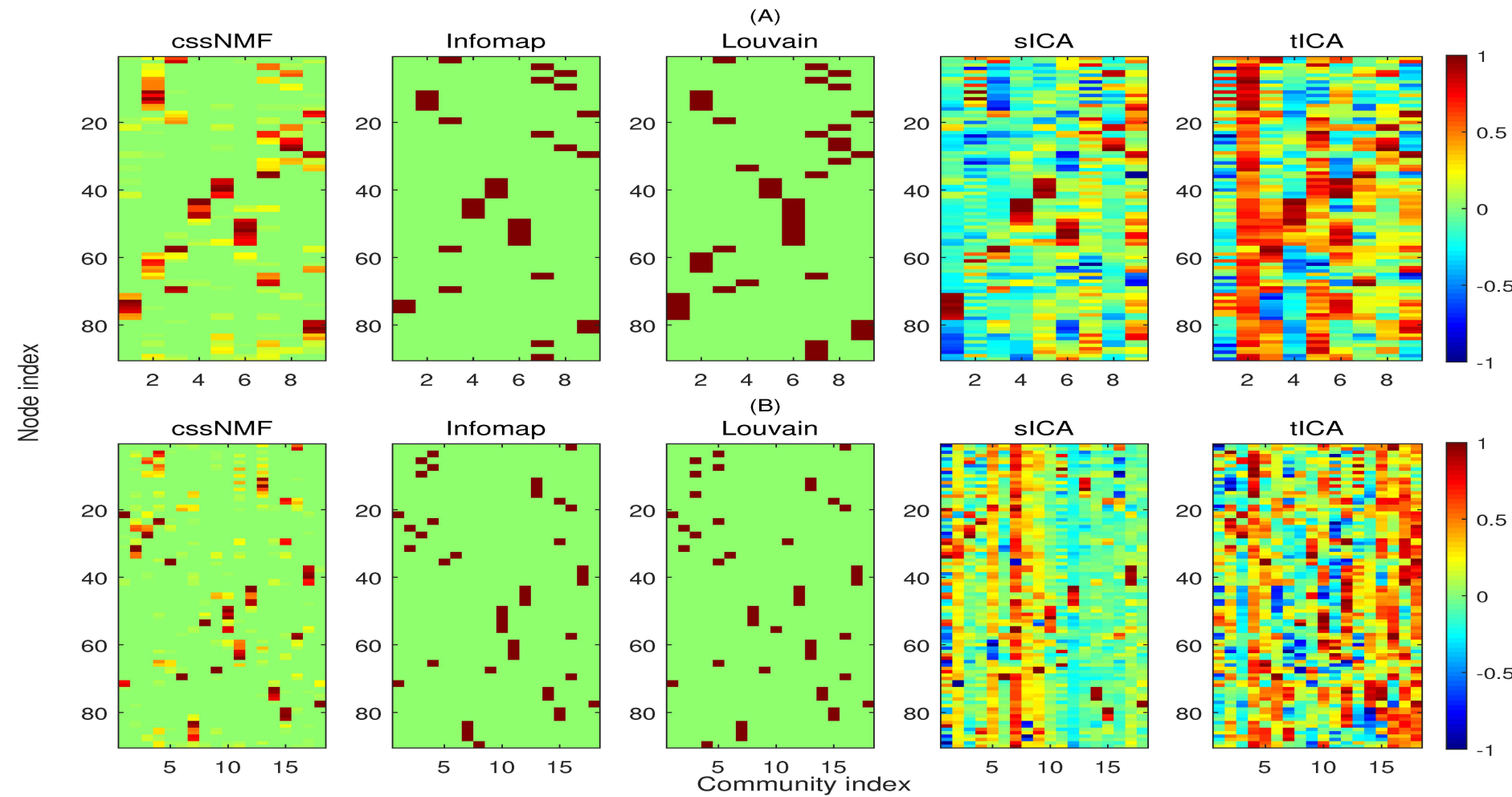

Figure 7: Results of the group-level communities identified by different methods on the real rs-fMRI data. (A) and (B) show the community structures identified by cssNMF, Infomap, Louvain method, sICA and tICA when $k=9$ and 18 respectively.

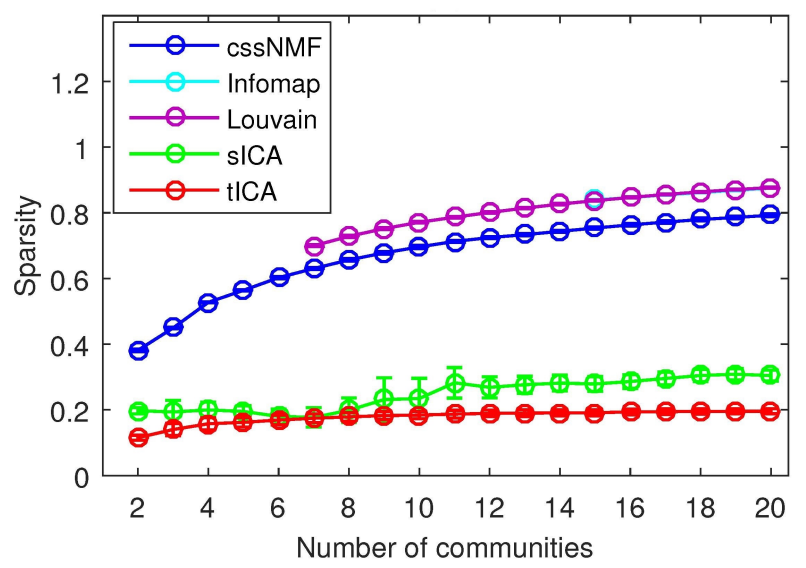

Figure 8: Overall sparsity of the group-level communities identified by cssNMF, Infomap, Louvain method, sICA and tICA with varying values of $k$ on the repeated split-half rsfMRI data. The error bars denote the standard deviation across all 200 datasets.
Furthermore, in order to compare cssNMF with the other four methods more intuitively in the group-level communities and further explore the neurophysiological incommunities represented by membership matrices in Figure 7 with $k=9$ onto the human brain models, as shown in Figure 9 Illustrations for the other four competing methods are provided in Figures S3-S6. In particular, Figure 7109 displays the communities derived by cssNMF based on the repeated split-half data with $\tau=0.1$, where the membership value of each node to each community indicates the median across all 200 datasets. The 9 communities derived by cssNMF shown here refer to basal ganglia (C1), fronto75 parietal $(\mathrm{C} 2)$, sensory-motor $(\mathrm{C} 3)$, primary visual $(\mathrm{C} 4)$, limbic (C5), extra-striate visual (C6), DMN (C7), orbital (C8) and insular-temporal/ACC (C9) networks separately, which are highly consistent with several well-recognized RSNs discovered by previous studies. Besides, as can be seen, the communities detected by cssNMF are also in line with the communities detected by the other four methods although to different extents (see Figures S3-S6). The quantitative measure of the similarity between cssNMF and 

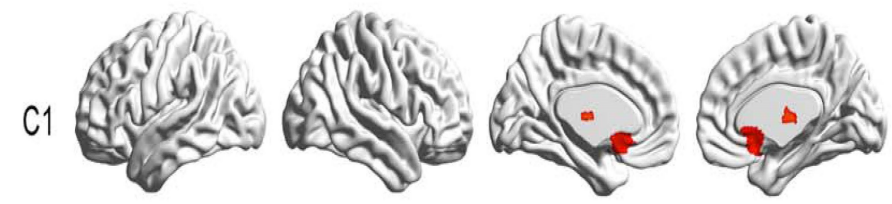

C2
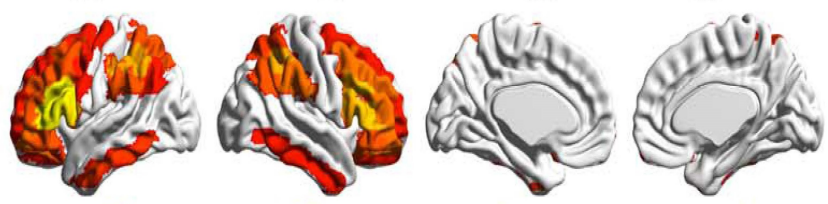

C3
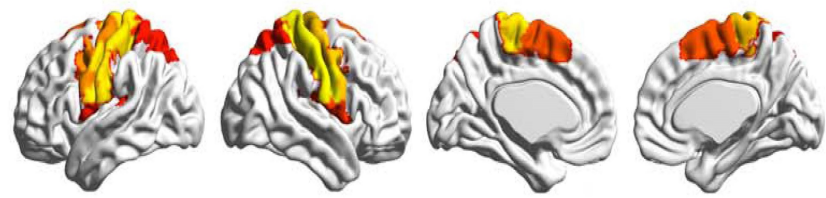

C4
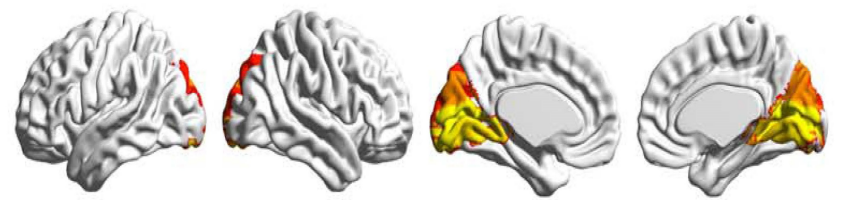

C5
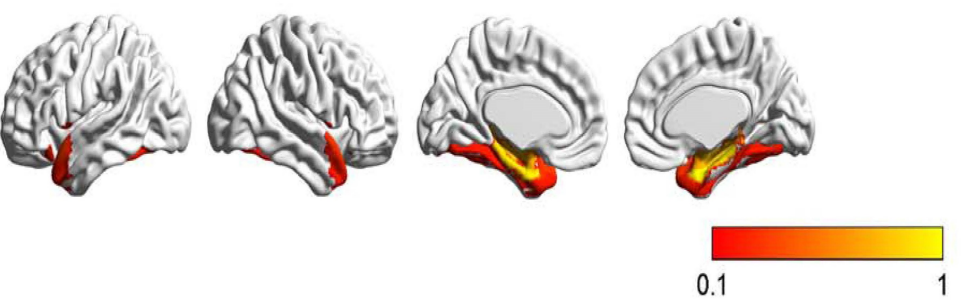
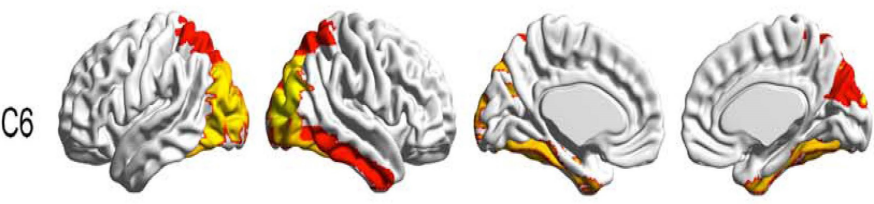

C7
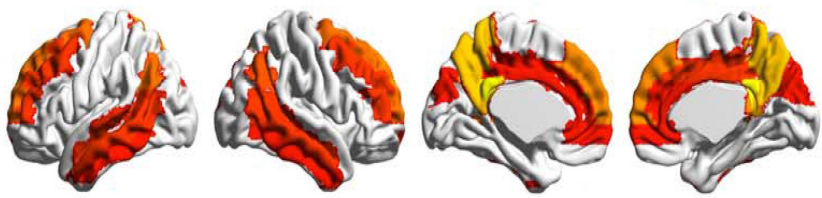

C8
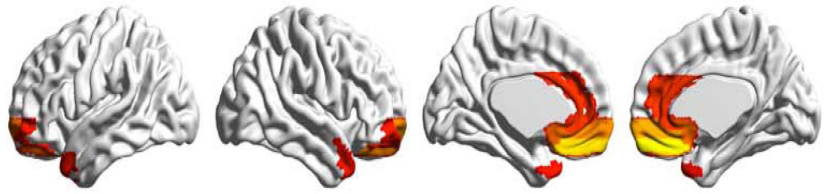

C9
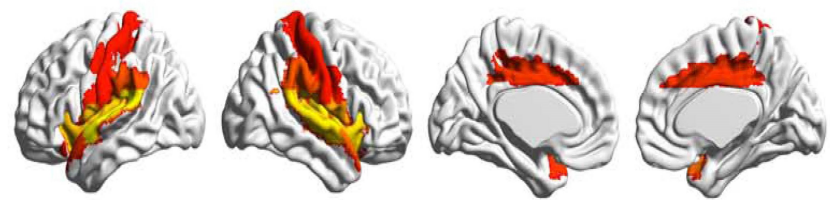

Figure 9: Illustration of the communities identified by ccsNMF on the human brain model. Results are derived with $k=9$ based on the repeated split-half datasets, where the weight of each node to each community is the median across all datasets. Communities $\mathrm{C} 1$ to $\mathrm{C} 9$ refer to basal ganglia, fronto-parietal, sensory motor, primary visual, limbic, extra-striate visual, DMN, orbital and insular-temporal/ACC networks respectively. The threshold $\tau=0.1$ for illustration. This figure is drawn by using BrainNet Viewer (Xia et al., 2013).
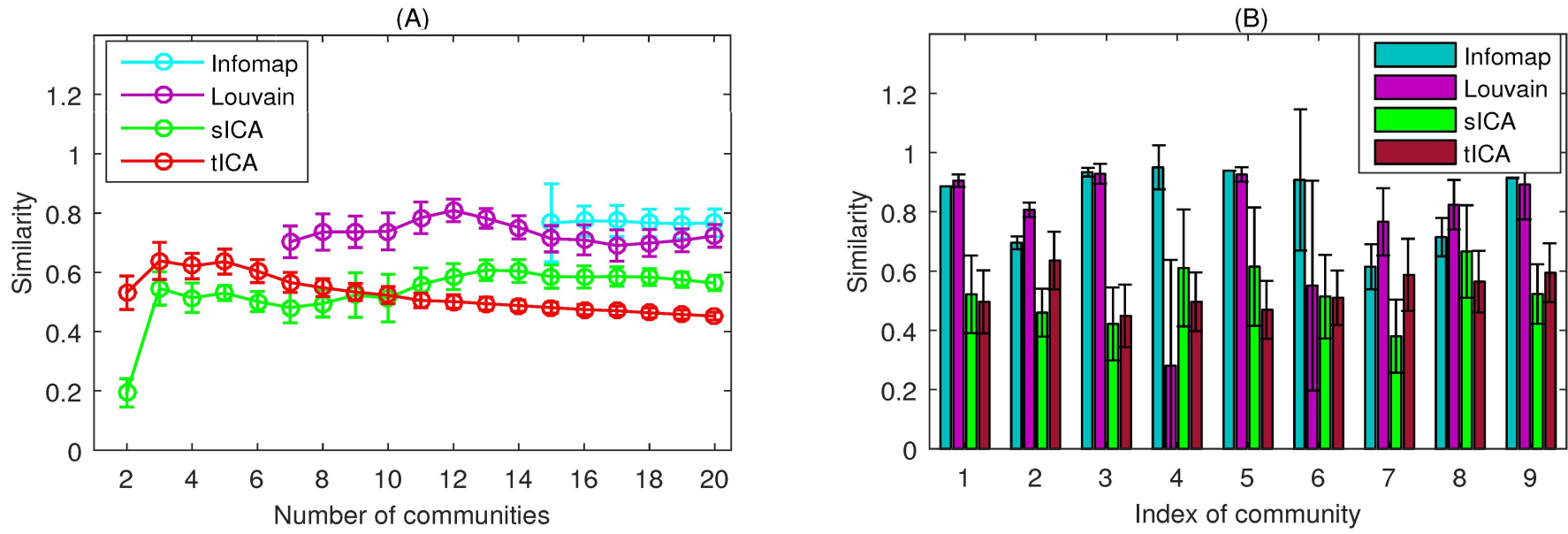

Figure 10: Similarity between cssNMF and the other four methods in group-level community structures on the repeated split-half rs-fMRI data. (A) Overall similarity between cssNMF and the other four methods including Infomap, Louvain method, sICA and tICA with varying values of $k$ in group-level community structures. (B) Similarity between cssNMF and the other four methods for individual communities separately. The error bars denote the standard deviation across all 200 datasets. 
the other four methods in the overall community structure separately. It reveals that Infomap and modularity optimization achieve a high similarity to cssNMF (from 0.699 to 0.809 ), whereas ICA methods achieve a moderate similarity around 0.5. Figure 10(B) further illustrates the similarity for individual communities separately. It can be 775 seen that these methods actually perform quite differently for individual communities. For example, for the primary visual network (C4), the similarity between Infomap and cssNMF reaches up to 0.950 , while modularity optimization only achieves a similarity of 0.281 . By scrutinizing this780 community in detail, we find that modularity optimization fails to separate primary visual network and extra-striate visual network and identifies the precuneus regions as a single network, which is known as a core part of DMN, while it has a high similarity to cssNMF in basal ganglia785 and sensory-motor networks. Besides, although both sICA and tICA achieve a similarity to cssNMF around 0.5 on average, for several communities sICA are more similar to cssNMF than tICA and vice versa for the rest communities.

We then further depicted the unique features of the communities detected by cssNMF by counting the number of the belonging communities for each region to illustrate community overlap, based on the median weights across all 200 datasets of the repeated split-half data with $\tau=0.1{ }^{795}$ as shown in Figure 11(A). It reveals that most regions are involved in more than one community and community overlap spans over frontal, parietal and temporal lobes. By contrast, regions related to sensori-motor and primary 755 visual participate in fewer communities. To more precisely ${ }^{80}$ pinpoint the overlapping nodes that take part in at least two communities, we used the following two stricter criteria to select the overlapping nodes based on all 200 subsets of the repeated split-half datasets. Firstly, as the overlapping nodes identified in each dataset might be different, the selected overlapping nodes are restricted to be identified consistently by more than $90 \%$ of all these datasets. Secondly, the multiple communities that they belong to are consistent across at least $90 \%$ of all these datasets. Considering the value of the threshold may affect the results ${ }^{81}$ of the identified overlapping nodes, we tested five different thresholds $\tau \in\{0.1,0.12,0.15,0.17,0.2\}$. The detailed description of all the identified nodes under each threshold and their belonging communities are shown in Tables S1-S3 in the supplementary material. All twenty-three overlapping nodes are drawn in Figure 11(B). Furthermore, we illustrate the distributions of the overlapping nodes identified under all values of $\tau$ over brain lobes and communities in Figures 11(C) and (D) respectively. The distribution of all overlapping nodes over brain lobes is consistent with the finding in Figure 11(A). Most of them are located in the parietal, frontal lobes and limbic structures (accounting for about $38 \%, 25.4 \%$ and $22.5 \%$ respectively of all overlapping nodes). The fronto-parietal network (C2) contains significantly more overlapping nodes than the other networks, which covers mainly the frontal and parietal lobes. The insular/ACC (C9), orbital networks (C8) and DMN (C7) also contain more than 20 overlapping nodes, while no overlapping nodes are found in the sensory-motor network (C3).

Next, we mainly evaluated the reproducibility of the group-level communities identified by all these methods under different values of $k$. Specifically, the result of crosssession reproducibility computed on two sessions of CoRR dataset is shown in Figure 12(A). On the whole, cssNMF achieves the highest cross-session reproducibility (from 0.798 to 0.999$)$ when $k$ varies from 2 to 16 . Infomap also achieves a high cross-session reproducibility (from 0.821 to 0.966 ) when $k \geq 17$, while tICA obtains the lowest score between 0.566 to 0.822 . It indicates that for the same cohort of participants cssNMF is able to derive highly stable communities across time. Furthermore, we evaluated the cross-subject reproducibility based on the repeated split-half datasets, as shown in Figure 12(B). Similarly, cssNMF still achieves a remarkably higher cross-subjects reproducibility (from 0.805 to 0.997 ) with $k$ varying from 2 to 15 , followed by modularity optimization, sICA and tICA. Infomap achieves the highest score when $k \geq 16$. In addition, another different dataset (Beijing_Zang dataset) was used by running the same procedure to compute the cross-data reproducibility, as shown in Figure 12(C). The cssNMF method achieves a score above 0.78 irrespective of the number of resulting communities (except at $k=2$ ) and the score reaches up to 0.953 . It substantially outperforms all the other methods, which means that the results derived by cssNMF are highly reproducible even across different datasets.

In addition to the group-level community structure, css- 
(A)

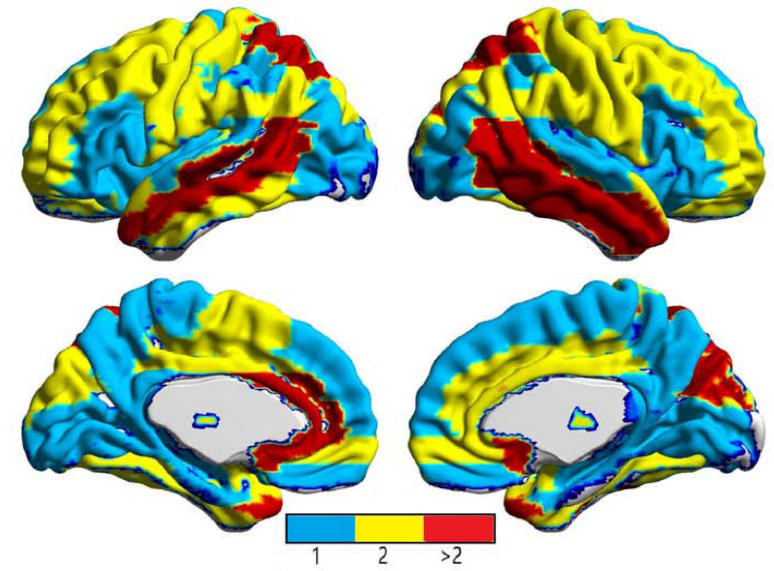

(C)

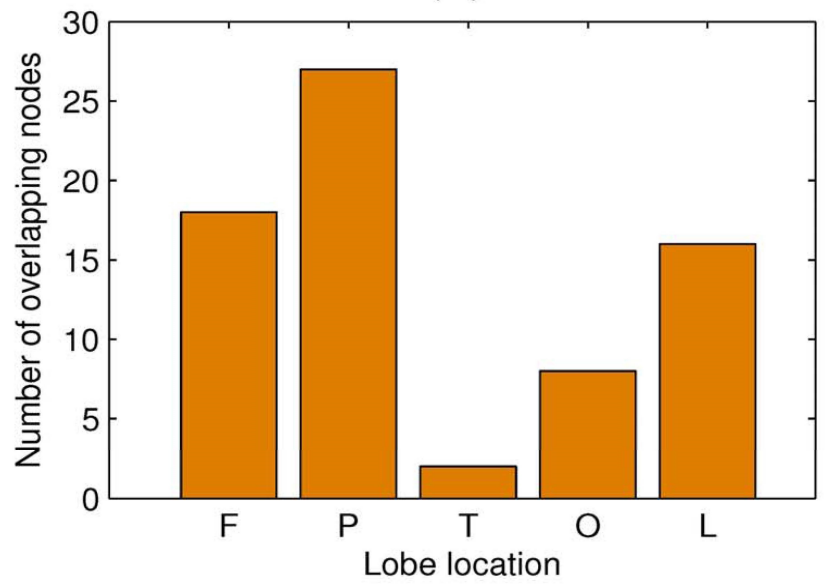

(B)

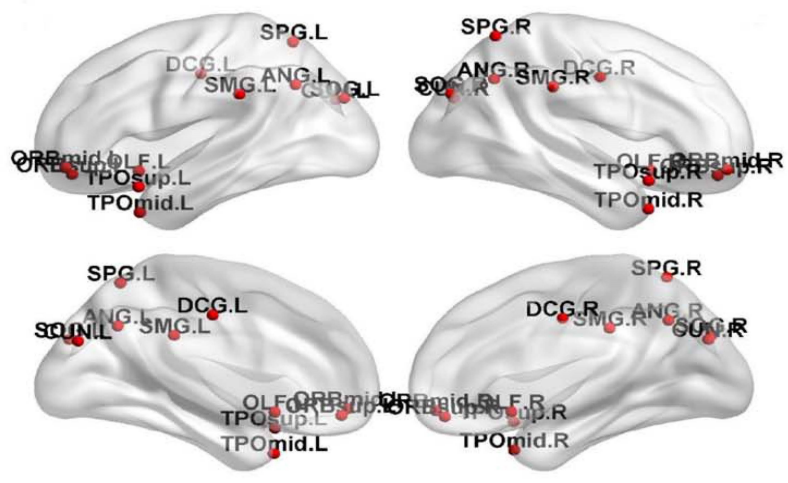

(D)

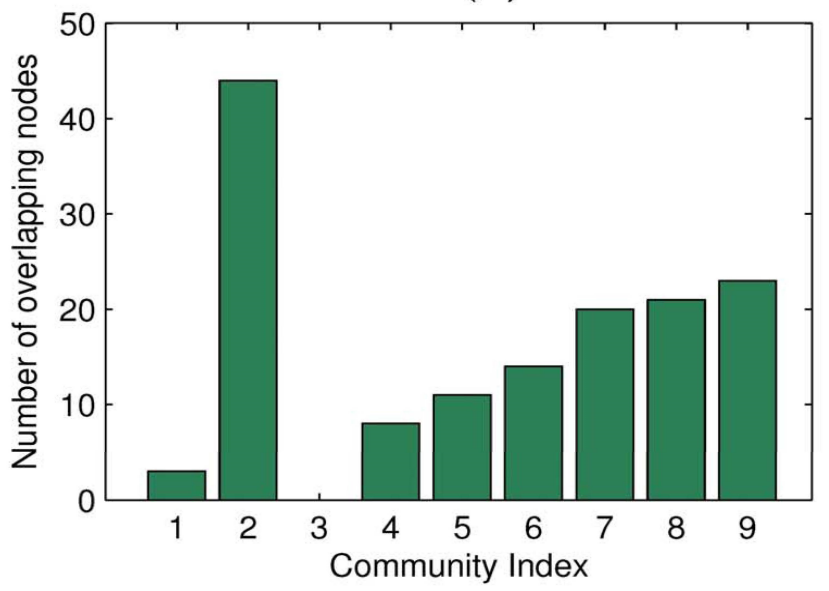

Figure 11: Illustrations and distributions of the overlapping nodes identified by ccsNMF. (A) The number of belonging communities is counted for each node based on the median weights across all 200 datasets of the repeated split-half data with $\tau=0.1$ and represented by different colours. (B) illustrations of the identified overlapping nodes. (C) Distribution of the overlapping nodes over different brain lobes, where the labels ' $\mathrm{F}$ ', 'P', 'T', 'O', 'L' refer to the frontal, parietal, temporal, occipital lobes and limbic structures respectively. (D) Distribution of the overlapping nodes over the 9 communities. 
(A)

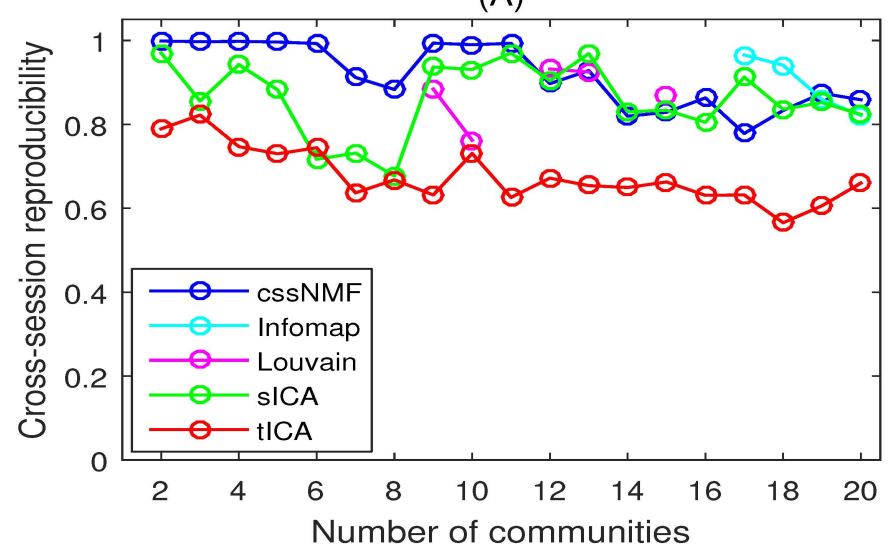

(B)

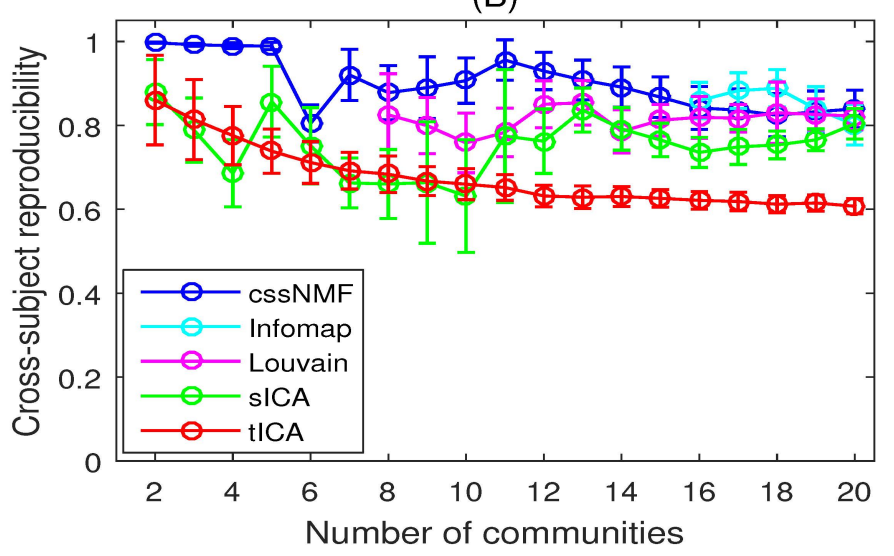

(C)

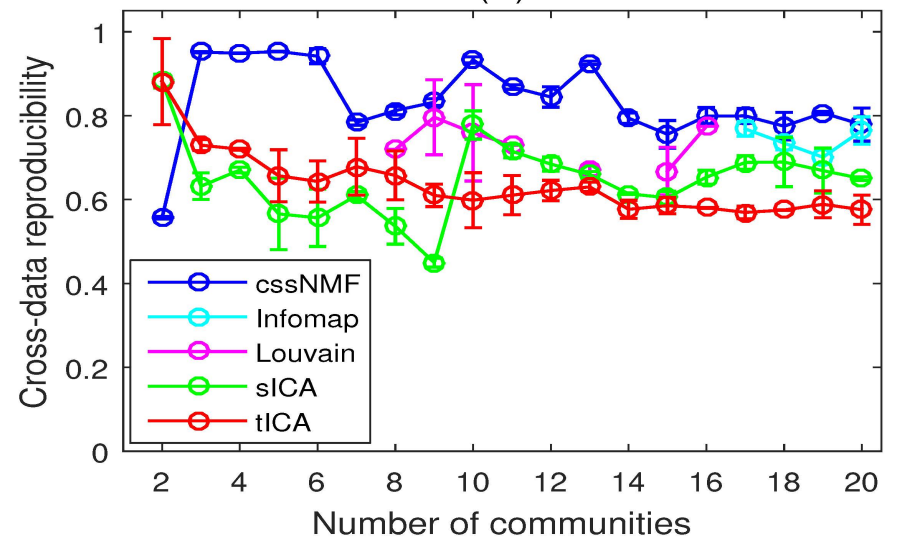

Figure 12: Reproducibility across sessions, subjects and datasets of the group-level community structures identified by cssNMF, Infomap, Louvain method, sICA and tICA on the real rs-fMRI data with varying number of communities. (A) Cross-session reproducibility of the group-level community structures identified on the CoRR data. (B) Mean cross-subject reproducibility of the group-level community structures identified across 100 split-half datasets of the CoRR data. (C) Cross-data reproducibility of the group-level community structures, which were identified on each session of the CoRR data against Beijing_Zang data separately and then averaged over the results on two sessions. 


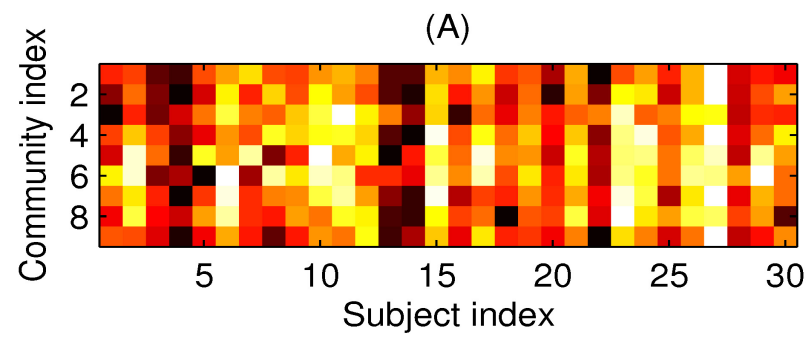

(C)

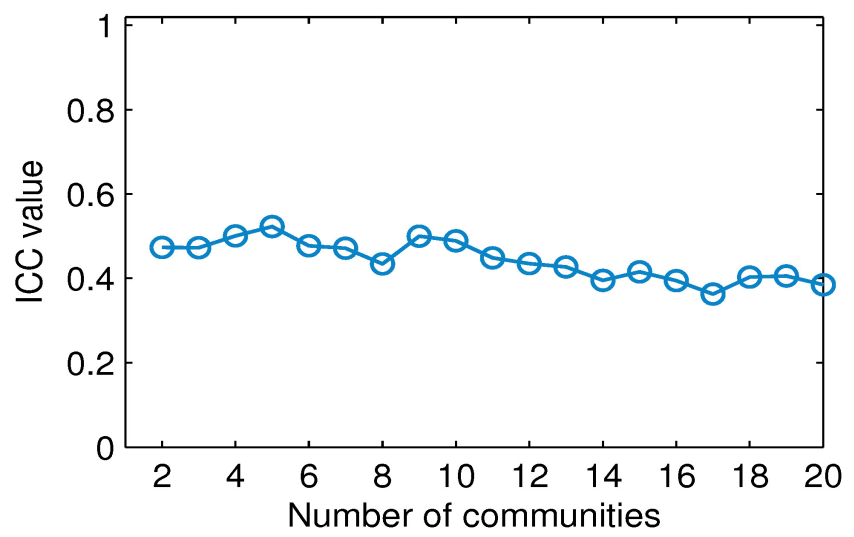

(B)

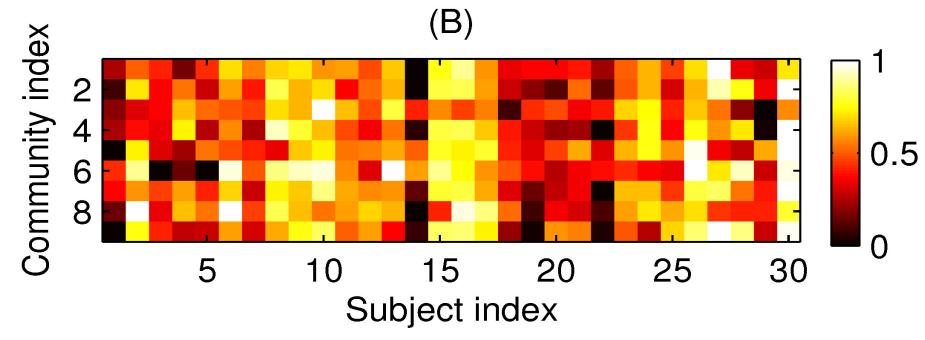

(D)

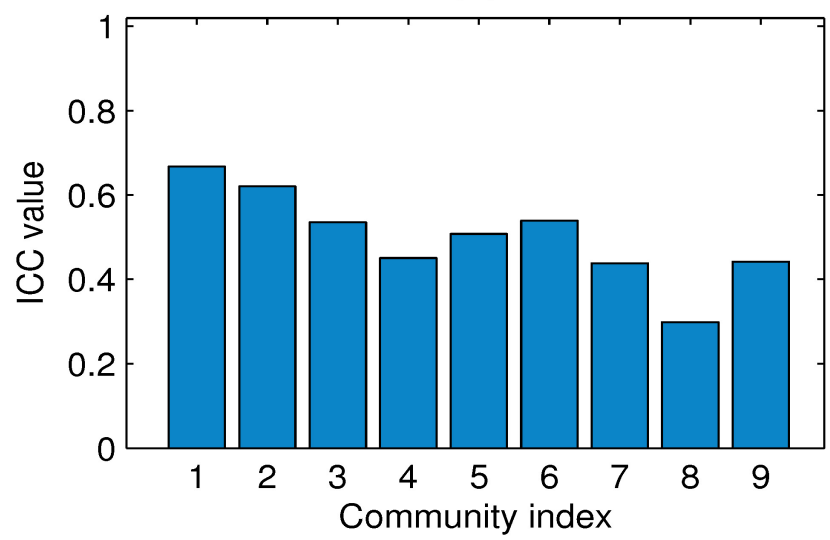

Figure 13: Results of the inter-subject variability identified by cssNMF on the CoRR dataset. (A) and (B) show the inter-subject variability in community strengths derived on session 1 and session 2 respectively with $k=9$. (C) Test-retest reliability of the inter-subject variability measured by the mean ICC index with varying values of $k$. (D) Test-retest reliability of each community measured by ICC with $k=9$.

are the most reliable $(0.632$ and 0.620 respectively within the substantial level) and community 8 performs worst (only 0.299 of the fair level ), while the rest communities exhibit a moderate level of reliability.

\section{Discussions}

In this paper, we propose cssNMF to identify the overlapping community structure in resting state brain functional networks, which is developed on the previous NMFbased methods. As a matter of fact, as a classic method which exists for almost two decades, only recently has NMF attracted great attention in brain imaging studies. Besides the studies mentioned in the introduction have applied NMF-based methods to fMRI data, NMF has also been adopted to find the common structural imaging patterns across individuals using structural magnetic resonance (sMR) data of the human brain (Sotiras et al. 2015). Moreover, significant alterations in the brain networks associated with attention deficit hyperactivity disorder (ADHD) have been revealed by applying NMF to multimodal data consisting of MRI, fMRI and phenotypic information Anderson et al. 2014). And in decoding single-trial M/EEG signals, NMF has been used to identify the temporal and spatial components simultaneously (Delis et al., 2016).

Two aspects are taken into account by cssNMF for com855 munity detection. Firstly, studying the group-level com- 
munity structure across subjects, especially the overlapping structure, can result in a better understanding of the mechanism of brain functioning. Secondly, capturing the individual differences could be useful to discriminate differ-

ent groups in particular for clinical practice. Several other905 approaches have been proposed by considering one or both aspects in recent studies. For example, Wu et al. (2011) investigated the structural overlapping brain network by using an algorithm based on k-cliques communities. $\mathrm{Du}$ and Fan (2013) proposed an improved group-ICA approach ${ }_{910}$ to identify both group-level and individual-level intrinsic networks based on fMRI time series directly. Yeo et al. (2014) have applied latent dirichlet allocation (LDA), which is closely related to NMF and ICA, to identify overlapping networks. Eavani et al. (2015) studied the overlapping ${ }_{15}$ brain network as well as the inter-subject variability focusing on negative correlations, while Najafi et al. (2016) detected the functional overlapping community structure by using a Bayesian model-based approach. By contrast, the proposed cssNMF approach uniquely identifies the920 group-level overlapping functional network organization by factorizing non-negative sparse association matrices derived by NASR and obtains the inter-subject variability in community strengths simultaneously without additional steps.

To our best knowledge, by proposing the cssNMF method, we for the first time have applied NMF to identify common functional connectivity patterns across individuals based on fMRI. Furthermore, it simultaneously retains the information of the inter-subject variability. Experimental 930 results show that the whole framework is capable of identifying the underlying network organization of the functional human brain accurately and consistently across varying time, subjects and datasets, while capturing the subtle individual differences in community strengths stably.

\subsection{Analysis on the simulated data}

In identifying the group-level community structure of the simulated dataset, cssNMF outperformed all the other methods in terms of similarity and accuracy, based on both the NASR-derived and the Pearson correlation-derived asso-940 ciation matrices. It may be owed to some unique properties of cssNMF. Firstly, as a community detection method, cssNMF inherits the advantages from the symmetric NMF, which naturally groups the highly functional-linked nodes into coherent parts with a plain explanation of the physical945 meaning that all these parts additively form the whole picture due to its non-negativity and in the meantime allows overlaps between different clusters. Secondly, its sparsity constraint in cssNMF further improves the results by assigning larger weights to the most coherent nodes and weights close to zero to the irrelative nodes within each community. As a result, it makes cssNMF rather insensitive to the selection of thresholds when obtaining the binary membership of communities. The performance of Infomap and modularity optimization, however, is more susceptible to the input association matrix, since they perform significantly better based on the NASR-derived association matrix than on the correlation-based one. Moreover, a common issue in both sICA and tICA is that they tend to obtain a denser result with negative values, although tICA shows a slight advantage over sICA due to its better ability to find overlapping community structure (Smith et al., 2012). For this simulated dataset, these negative values are especially difficult to interpret, since the ground-truth has shown that the communities are not anti-correlated with each other. Furthermore, cssNMF also exhibited a remarkable ability to capture the inter-subject variability in community strengths on both type of association matrices, by reason of the collective way used to preserve individual specified information. Besides, the accurate construction of the brain functional network by NASR, which provides a solid basis for cssNMF, is also an important contributing factor to its superior performance, since the accuracy approached as high as $100 \%$ for the NASR method. To sum up, results on simulated dataset suggest that the cssNMF approach is able to identify the group-level community structure and the individual differences in community strengths effectively and accurately.

\subsection{Analysis on the real rs-fMRI data}

The quantitative analysis on the real rs-fMRI data mainly focuses on the reproducibility of the community structure and the test-retest reliability of the inter-subject variability. In identifying the group-level community structure, cssNMF achieved higher reproducibility than the other methods in all three reproducibility measures including cross-session, cross-subject and cross-data basically in most cases. It means that the community structures identified by cssNMF are considerably consistent across varying sessions, subjects and even different datasets. The sparsity constraint in cssNMF may account for its robustness, since 
it largely reduces the uncertainty of the results whereby enforcing cssNMF to retain only the most relevant information and exclude the others. There are also some interesting findings regarding the inter-subject variability. The high functional networks under various voxel-wise metrics. In addition, it has been reported by Finn et al. (2015) that the fronto-parietal network is most distinct across individuals, which may implicate a high inter-subject variability that will contribute to a higher ICC.

${ }_{980}$ More importantly, the overlapping communities derived ${ }^{1025}$ by cssNMF are not only highly reproducible but also neurophysiologically meaningful. In this experiment, we adopted a relatively coarse node definition of 90 ROIs so as to reduce the computational burden of the construction of the whole-brain network. It is suggested that node defini- ${ }^{-103}$ tions of different sizes or degrees of functional homogeneity within ROIs may affect the identified network organization (Poldrack, 2007). Here, we have also performed the cssNMF based on a more refined atlas of 264 ROIs proposed this atlas although results may differ in some details, as shown in Figures S7. Actually, myriads of studies have detected RSNs on different scales of the brain by using various methods. Yeo et al. (2011) proposed a 7-network parcellation based on more than 1000 ROI vertices. And Van den Heuvel and Pol (2010) reported eight most consistent RSNs across different studies using clustering or ICA-based methods. These RSNs mainly include the DMN, fronto-parietal network, primary and extra-striate visual network, insular/temporal ACC network and so on, which are largely in accordance with the nine communities identified by cssNMF in this paper.

Furthermore, the overlapping communities derived by cssNMF are comparable to the communities derived by the other methods. On average, most communities detected by cssNMF also appear in the results derived by both Infomap and modularity optimization with a high similarity around 0.8. However, the most prominent difference is that only cssNMF is able to capture the widely-spread community overlap across frontal, parietal and temporal regions in the association cortex. Moreover, sICA and tICA exhibit a great diversity in their similarity with cssNMF for different communities. Basically, tICA shows higher similarity with cssNMF than sICA for communities containing more overlapping nodes, such as the fronto-parietal network and DMN, due to its better ability to identify overlapping networks, while communities containing fewer overlapping nodes such as primary visual network identified by cssNMF resemble those by sICA more than by tICA. In other words, the cssNMF-identified community structure is partially supported by sICA and partially by tICA with little overlap and can not be replaced by each other. It could be easily understood because these matrix factorization-based methods work under different assumptions, thus reflecting various facets of the human brain network structure. In this sense, cssNMF at least provides a complementary approach to investigate the overlapping community structure of the human brain. Furthermore, although sICA and tICA are able to capture the overlapping networks as well, the main drawback is that they derive very dense networks, where few nodes have zero weights, thus making the results lack a direct interpretation.

Moreover, some interesting features were also found in the overlapping nodes. The identified overlapping nodes occur widely in the frontal and parietal brain regions, which 
are closely related to the fronto-parietal network. Similar results that some most flexible hubs implicated in cognitive control are included in the fronto-parietal network have been found by several recent studies (Cole et al. 2013.

van den Heuvel and Sporns, 2013; Zanto and Gazzaleyloss 2013). The bilateral angular gyri which play an important role in both DMN and the fronto-parietal network have been revealed to serve multiple functions that are related to various tasks (Seghier, 2013). The superior and middle bilateral temporal poles that are in charge of sensory integration have been classified as both structure and func- ${ }^{1090}$ tional cores in (Pascual et al. 2013). The superior parietal regions have also been recognized as an important hub to mediate information from distributed regions (Hwang et al. 2012). These overlapping nodes indicate that the corresponding brain regions may play flexible roles in executing ${ }^{1095}$ various brain functions and are responsible for information integration. On the other hand, the sensory-motor and primary visual network that have fewer overlapping nodes are relatively isolated, and these regions have been reported to have high local connectivity (Buckner and Krienen, 2013$)^{100}$ Yeo et al. 2014).

\subsection{Future work and limitations}

Unlike conventional community detection methods, such as Infomap and modularity optimization, the cssNMF method derives a continuous solution of the community structure, thus allowing community overlap. On the one hand, it provides more than just binary clustering assignments, since it indicates how the nodes are involved in each community via membership values. On the other hand, an inevitable limitation lying in cssNMF per se alongside with its merits is that a threshold is needed if one wants to obtain the binary clustering assignments. In this case, the threshold applied should be at least larger than the minimum of the maximum membership values of all nodes, so that each node is ensured to belong to at least one community.

Another limitation of cssNMF is that the performance of cssNMF depends on its initialization to some extent, although not as heavily as clustering algorithms such as Kmeans. In this paper, the cssNMF algorithm is initialized ${ }_{120}$ randomly, which is the most popular and simple initialization strategy for NMF methods. In fact, there are other useful initialization techniques for NMF methods, such as spherical k-means (Wild et al. 2004) and nonnegative double singular value decomposition (NNDSVD) (Boutsidis and Gallopoulos, 2008). These alternative strategies may lead to faster convergence of the algorithm. However, they still do not provide guarantees for the quality of the obtained solutions by cssNMF. In our experiment, we launched multiple runs with different initializations for cssNMF to select the best result, which is a simple way in practice to alleviate the influence of random initializations on the quality of solutions.

Moreover, further improvements could be made on cssNMF regarding its sparsity constraint. In this paper, we adopt the widely used $\ell_{1}$-norm to enforce a global sparsity on the obtained community structure. More structured forms of sparsity could be applied to cssNMF, by incorporating the techniques provided by recent studies in various domains. For example, an $\ell_{1,2}$-norm used in sparse learning (Kong et al., 2014) could be used to enforce sparsity at the level of intra-community. Besides, it has been shown that NMF methods could benefit from applying a more natural sparsity constraint $\ell_{0}$-pseudo norm (Peharz and Pernkopf, 2012).

Besides, the ability of cssNMF for capturing the individual differences in community strengths may be beneficial for studying fMRI data from different groups. In other words, the individually specified information on community strengths, which may reflect changes in network organization, could be viewed as discriminatory features to distinguish different brain states or identify brain disorders. Thus, we aim to apply cssNMF on such datasets in our future work, which may contribute to the early diagnosis of brain disorders. Before that, the test-retest reliability of cssNMF in capturing such individual differences could be further tested by using different data preprocessing methods or analysis approaches such as network construction to lower the intra-subject variability and by using different datasets containing more heterogeneous subjects with larger inter-subject variability.

Finally, using the brain functional imaging data alone may not be enough to gain a comprehensive understanding of the brain functional organization. A multimodal way of combining the brain's structural information with functional data may provide more knowledge about the underlying substrates and guide us to a deeper understanding of the brain network organization. 


\section{Acknowledgments}

The authors would like to thank Dr. Xinian Zuo for providing the CoRR dataset and his help in data preprocessing and analysis. This work was supported in part by the National Basic Research Program of China under Grant 2015CB351704, the Key Research and Development Plan (Industry Foresight and Common Key Technology) $\overline{1}_{200}$ Key Project of Jiangsu Province under Grant BE2017007-3, the National Natural Science Foundation of China under Grants 61773114 and 61375118, and the China Scholarship Council Fund under Grant 201603780037. The authors ${ }_{205}$ would also like to thank our anonymous reviewers for their constructive comments and suggestions that have considerably improved this manuscript.

\section{Disclosure/conflict-of-interest statement}

We wish to confirm that there are no known conflicts of interest associated with this manuscript and there has been ${ }^{1215}$ no significant financial support for this work that could have influenced its outcome.

\section{Role of the funding source}

The research was conducted in the absence of any commercial or financial relationships that could be construed as a potential conflict of interest.

\section{References}

Anderson, A., Douglas, P.K., Kerr, W.T., Haynes, V.S., Yuille, A.L., Xie, J., Wu, Y.N., Brown, J.A., Cohen, M.S., 2014. Non-negative matrix factorization of multimodal MRI, fMRI and phenotypic data reveals differential changes in default mode subnetworks in 1. ADHD. Neuroimage 102, 207-219. doi $10.1016 / j \cdot$ neuroimage 2013.12.015

Biswal, B., Yetkin, F.Z., Haughton, V.M., Hyde, J.S., 1995. Functional connectivity in the motor cortex of resting human brain

n using echo-planar MRI. Magn. Reson. Med. 34, 537-541. doi 10 1002/mrm.1910340409

Biswal, B.B., Mennes, M., Zuo, X.N., Gohel, S., Kelly, C., Smith, S.M., Beckmann, C.F., Adelstein, J.S., Buckner, R.L., Colcombe, S., Dogonowski, A.M., Ernst, M., Fair, D., Hampson, M., Hoptman, M.J., Hyde, J.S., Kiviniemi, V.J., Kötter, R., Li, S.J., Lin, C.P., Lowe, M.J., Mackay, C., Madden, D.J., Madsen, K.H., Margulies, D.S., Mayberg, H.S., McMahon, K., Monk, C.S., Mostofsky, S.H., Nagel, B.J., Pekar, J.J., Peltier, S.J., Petersen, S.E., Riedl, V., Rombouts, S.A.R.B., Rypma, B., Schlaggar, B.L., Schmidt, S., Seidler, R.D., Siegle, G.J., Sorg, C., Teng, G.J., Veijola, J., Villringer, A., Walter, M., Wang, L., Weng, X.C., Whitfield-Gabrieli, S., Williamson, P., Windischberger, C., Zang, Y.F., Zhang, H.Y., Castellanos, F.X., Milham, M.P., 2010. Toward discovery science of human brain function. Proc. Natl. Acad. Sci. 107, 4734-4739. doi 10.1073/pnas.0911855107

Boutsidis, C., Gallopoulos, E., 2008. SVD based initialization: A head start for nonnegative matrix factorization. Pattern Recognit. 41, 1350-1362. doi 10.1016/j·patcog.2007.09.010

Boyd, S., Parikh, N., Chu, E., Peleato, B., Eckstein, J., 2011. Distributed optimization and statistical learning via the alternating direction method of multipliers. Found.Trends(R) Mach. Learn. 3, 1-122. doi $10.1561 / 2200000016$

Braun, U., Plichta, M.M., Esslinger, C., Sauer, C., Haddad, L., Grimm, O., Mier, D., Mohnke, S., Heinz, A., Erk, S., Walter, H., Seiferth, N., Kirsch, P., Meyer-Lindenberg, A., 2012. Test-retest reliability of resting-state connectivity network characteristics using fMRI and graph theoretical measures. Neuroimage 59, 1404-1412. doi 10.1016/j.neuroimage.2011.08.044.

Buckner, R.L., Krienen, F.M., 2013. The evolution of distributed association networks in the human brain. Trends Cogn. Sci. 17, 648-665. doi 10.1016/j.tics.2013.09.017

Buckner, R.L., Krienen, F.M., Yeo, B.T., 2013. Opportunities and limitations of intrinsic functional connectivity MRI. Nat. Neurosci. 16, 832-837. doi 10.1038/nn.3423.

Bullmore, E., Sporns, O., 2009. Complex brain networks: Graph theoretical analysis of structural and functional systems. Nat. Rev. Neurosci. 10, 186-198. doi 10.1038/nrn2575

Bullmore, E., Sporns, O., 2012. The economy of brain network

口 organization. Nat. Rev. Neurosci. 13, 336-349. doi 10.1038/ nrn3214. 
Cao, H., Plichta, M.M., Schäfer, A., Haddad, L., Grimm, O., Schneider, M., Esslinger, C., Kirsch, P., Meyer-Lindenberg, A., Tost, H., 2014. Test-retest reliability of fMRI-based graph theoretical properties during working memory, emotion processing, and resting

1. state. Neuroimage $84,888-900$. doi $10.1016 / j$.neuroimage. 2013 i 80 09.013

Chen, B., Xu, T., Zhou, C., Wang, L., Yang, N., Wang, Z., Dong, H.M., Yang, Z., Zang, Y.F., Zuo, X.N., Weng, X.C., 2015. Individual variability and test-retest reliability revealed by ten repeated resting-state brain scans over one month. PloS ONE 10, e0144963285 doi 10.1371/journal. pone.0144963.

Cole, M.W., Reynolds, J.R., Power, J.D., Repovs, G., Anticevic, A., Braver, T.S., 2013. Multi-task connectivity reveals flexible hubs for adaptive task control. Nat. Neurosci. 16, 1348-1355. doi $10.1038 / \mathrm{nn} .3470$

1290

De Luca, M., Beckmann, C., De Stefano, N., Matthews, P., Smith, S.M., 2006. fMRI resting state networks define distinct modes of long-distance interactions in the human brain. Neuroimage 29, 1359-1367. doi 10.1016/j.neuroimage.2005.08.035.

Delis, I., Onken, A., Schyns, P.G., Panzeri, S., Philiastides, M.G.1295 2016. Space-by-time decomposition for single-trial decoding of

M/EEG activity. Neuroimage 133, 504-515. doi 10.1016/j neuroimage.2016.03.043

Ding, C.H., He, X., Simon, H.D., 2005. On the equivalence of nonnegative matrix factorization and spectral clustering, in: Proceedings of 300 the 5th SIAM International Conference on Data Mining, SIAM. pp. 606-610. doi http://dx.doi.org/10.1137/1.9781611972757.70

Du, Y., Fan, Y., 2013. Group information guided ICA for fMRI data I analysis. Neuroimage 69, 157-197. doi 10.1016/j.neuroimage. 2012.11 .008

Eavani, H., Satterthwaite, T.D., Filipovych, R., Gur, R.E., Gur, R.C., Davatzikos, C., 2015. Identifying sparse connectivity patterns in the brain using resting-state fMRI. Neuroimage 105, 286-299. doi $10.1016 / \mathrm{j}$.neuroimage.2014.09.058

Ferdowsi, S., Abolghasemi, V., Makkiabadi, B., Sanei, S., $2011_{1310}$ A new spatially constrained NMF with application to fMRI, in: Engineering in Medicine and Biology Society, EMBC, 2011 Annual

II International Conference of the IEEE, IEEE. pp. 5052-5055. doi 10 1109/IEMBS. 2011.6091251

Ferdowsi, S., Abolghasemi, V., Sanei, S., 2010. A constrained NMF315 algorithm for BOLD detection in fMRI, in: Machine Learning for Signal Processing (MLSP), 2010 IEEE International Workshop on, IEEE. pp. 77-82. doi 10.1109/MLSP. 2010.5589216.

Finn, E.S., Shen, X., Scheinost, D., Rosenberg, M.D., Huang, J., Chun, M.M., Papademetris, X., Constable, R.T., 2015. Functional 320 connectome fingerprinting: Identifying individuals using patterns

of brain connectivity. Nat. Neurosci. 18, 1664-1671. doi 10.1038/ nn. 4135

Fransson, P., 2005. Spontaneous low-frequency BOLD signal fluctuations: An fMRI investigation of the resting-state default mode 325 of brain function hypothesis. Hum. Brain mapp. 26, 15-29. doi $10.1002 / \mathrm{hbm} .20113$

Friston, K.J., Harrison, L., Penny, W., 2003. Dynamic causal mod口 elling. Neuroimage 19, 1273-1302. doi 10.1016/S1053-8119(03) 00202-7

Grave, E., Obozinski, G.R., Bach, F.R., 2011. Trace lasso: A trace norm regularization for correlated designs, in: Proceedings of
Advances in Neural Information Processing Systems, MIT Press. pp. 2187-2195.

Greicius, M.D., Krasnow, B., Reiss, A.L., Menon, V., 2003. Functional connectivity in the resting brain: A network analysis of the default mode hypothesis. Proc. Natl. Acad. Sci. 100, 253-258. doi 10 1073/pnas.0135058100

Greicius, M.D., Srivastava, G., Reiss, A.L., Menon, V., 2004. Defaultmode network activity distinguishes Alzheimer's disease from healthy aging: Evidence from functional MRI. Proc. Natl. Acad. Sci. 101, 4637-4642. doi 10.1073/pnas.0308627101

von dem Hagen, E.A., Stoyanova, R.S., Baron-Cohen, S., Calder, A.J., 2012. Reduced functional connectivity within and between 'social' resting state networks in autism spectrum conditions. Soc. Cogn. Affect. Neurosci. 8, 694-701. doi 10.1093/scan/nss053

van den Heuvel, M.P., Sporns, O., 2013. Network hubs in the human

1) brain. Trends Cogn. Sci. 17, 683-696. doi 10.1016/j.tics.2013. 09.012

Hoyer, P.O., 2002. Non-negative sparse coding, in: Proceedings of the 12th IEEE Workshop on Neural Networks for Signal Processing, IEEE press. pp. 557-565. doi http://dx.doi.org/10.1109/NNSP. 2002.1030067

Hoyer, P.O., 2004. Non-negative matrix factorization with sparseness constraints. J. Mach. Learn. Res. 5, 1457-1469.

Huang, S., Li, J., Sun, L., Ye, J., Fleisher, A., Wu, T., Chen, K., Reiman, E., Initiative, A.D.N., 2010. Learning brain connectivity of Alzheimer's disease by sparse inverse covariance estiman tion. Neuroimage 50, 935-949. doi https://doi.org/10.1016/j neuroimage.2009.12.120

Hwang, K., Hallquist, M.N., Luna, B., 2012. The development of hub architecture in the human functional brain network. Cereb. Cortex 23, 2380-2393. doi/https://doi.org/10.1093/cercor/bhs227

Hyvärinen, A., 1999. Fast and robust fixed-point algorithms for independent component analysis. IEEE Trans. Neural Netw. 10, 626-634.

Kong, D., Fujimaki, R., Liu, J., Nie, F., Ding, C., 2014. Exclusive feature learning on arbitrary structures via $\ell_{1,2}$-norm, in: Advances in Neural Information Processing Systems, pp. 1655-1663.

Kuang, D., Ding, C., Park, H., 2012. Symmetric nonnegative matrix factorization for graph clustering, in: Proceedings of the 2012 SIAM international conference on data mining, SIAM. pp. 106117. doi $10.1137 / 1.9781611972825 .10$

Landis, J.R., Koch, G.G., 1977. The measurement of observer agreement for categorical data. Biometrics 33, 159-174.

Lee, D.D., Seung, H.S., 1999. Learning the parts of objects by nona negative matrix factorization. Nature 401, 788-791. doi 10.1038/ 44565

Lee, D.D., Seung, H.S., 2001. Algorithms for non-negative matrix factorization, in: Proceedings of Advances in Neural Information Processing Systems, MIT Press. pp. 556-562.

Li, X., Hu, Z., Wang, H., 2016a. Overlapping community structure detection of brain functional network using non-negative matrix factorization, in: Proceedings of the 23rd International Conference 1 on Neural Information Processing, Springer. pp. 140-147. doi 10. 1007/978-3-319-46675-0_16

Li, X., Hu, Z., Wang, H., 2016b. Sparse-network based framework for detecting the overlapping community structure of brain functional network, in: Proceedings of Advances in Brain Inspired Cognitive 

32

Li, X., Wang, H., 2015. Identification of functional networks in resting state fMRI data using adaptive sparse representation and affinity

1 propagation clustering. Front. Neurosci. 9. doi 10.3389/fnins 2015.00383

Lovász, L., Plummer, M.D., 1986. Matching theory. Ann. Discret. Math 29

Lu, C., Feng, J., Lin, Z., Yan, S., 2013. Correlation adaptive subspace segmentation by trace lasso, in: Proceedings of the 2013 IEEE International Conference on Computer Vision, IEEE press. рpно0 1345-1352. doi http://dx.doi.org/10.1109/ICCV.2013.170

Najafi, M., McMenamin, B.W., Simon, J.Z., Pessoa, L., 2016. Overlapping communities reveal rich structure in large-scale brain networks during rest and task conditions. Neuroimage 135, 92-106. doi $10.1016 / j$.neuroimage.2016.04.054

Newman, M.E., 2006. Modularity and community structure in net-

1 works. Proc. Natl. Acad. Sci. 103, 8577-8582. doi 10.1073/pnas 0601602103

O'Reilly, J.X., Beckmann, C.F., Tomassini, V., Ramnani, N., Johansen-Berg, H., 2010. Distinct and overlapping functional zones410 in the cerebellum defined by resting state functional connectivity. Cereb. Cortex 20, 953-965. doi 10.1093/cercor/bhp157.

Pascual, B., Masdeu, J.C., Hollenbeck, M., Makris, N., Insausti, R., Ding, S.L., Dickerson, B.C., 2013. Large-scale brain networks of the human left temporal pole: A functional connectivity MRI415

1. study. Cereb. Cortex 25, 680-702. doi https://doi.org/10.1093/ cercor/bht260

Peharz, R., Pernkopf, F., 2012. Sparse nonnegative matrix factoriza-

a tion with $\ell_{0}$-constraints. Neurocomputing 80,38-46. doi 10.1016/ j.neucom.2011.09.024

Pessoa, L., 2014. Understanding brain networks and brain organization. Phys. Life Rev. 11, 400-435. doi $10.1016 / j \cdot p l r e v .2014 .03$ 005.

Poldrack, R.A., 2007. Region of interest analysis for fMRI. Soc. Cogn. Affect. Neurosci. 2, 67-70. doi 10.1093/scan/nsm006

Potluru, V.K., Calhoun, V.D., 2008. Group learning using contrast NMF: Application to functional and structural MRI of schizophrenia, in: Circuits and Systems, 2008. ISCAS 2008. IEEE Interna-

口 tional Symposium on, IEEE. pp. 1336-1339. doi 10.1109/ISCAS 2008.4541673

Power, J.D., Cohen, A.L., Nelson, S.M., Wig, G.S., Barnes, K.A., Church, J.A., Vogel, A.C., Laumann, T.O., Miezin, F.M., Schlaggar, B.L., Petersen, S.E., 2011. Functional network organization

口 of the human brain. Neuron 72,665-678. doi 10.1016/j.neuron. 2011.09.006

Rosvall, M., Bergstrom, C.T., 2008. Maps of random walks on complex networks reveal community structure. Proc. Natl. Acad. Sci. 105, 1118-1123. doi 10.1073/pnas.0706851105

Rubinov, M., Sporns, O., 2010. Complex network measures of brain connectivity: uses and interpretations. Neuroimage 52, 1059-1069 doi $10.1016 / j$.neuroimage.2009.10.003

Seghier, M.L., 2013. The angular gyrus multiple functions and

n multiple subdivisions. The Neuroscientist 19, 43-61. doi https: //doi.org/10.1177/1073858412440596

Shehzad, Z., Kelly, A.C., Reiss, P.T., Gee, D.G., Gotimer, K., Uddim445 L.Q., Lee, S.H., Margulies, D.S., Roy, A.K., Biswal, B.B., Petkova,
E., Castellanos, F.X., Milham, M.P., 2009. The resting brain: 1. Unconstrained yet reliable. Cereb. Cortex 19, 2209-2229. doi 10 . 1093/cercor/bhn256

Shrout, P.E., Fleiss, J.L., 1979. Intraclass correlations: Uses in 1. assessing rater reliability. Psychol. Bull. 86, 420-428. doi 10.1037/ 0033-2909.86.2.420

Smith, S.M., Miller, K.L., Moeller, S., Xu, J., Auerbach, E.J., Woolrich, M.W., Beckmann, C.F., Jenkinson, M., Andersson, J., Glasser, M.F., Van Essen, D.C., Feinberg, D.A., Yacoubb, E.S., Ugurbilb, K., 2012. Temporally-independent functional modes of spontaneous brain activity. Proc. Natl. Acad. Sci. 109, 3131-3136. doi $10.1073 /$ pnas.1121329109

Smith, S.M., Miller, K.L., Salimi-Khorshidi, G., Webster, M., Beckmann, C.F., Nichols, T.E., Ramsey, J.D., Woolrich, M.W., 2011. Network modelling methods for fMRI. Neuroimage 54, 875-891. doi $10.1016 / j$.neuroimage.2010.08.063.

Sotiras, A., Resnick, S.M., Davatzikos, C., 2015. Finding imaging patterns of structural covariance via non-negative matrix factorization. Neuroimage 108, 1-16. doi 10.1016/j.neuroimage.2014.11.045

Sporns, O., 2010. Networks of the Brain. MIT press.

Sporns, O., 2013. Network attributes for segregation and integration in the human brain. Current opinion in neurobiology 23, 162-171. doi $10.1016 / j$. conb.2012.11.015

Sporns, O., 2014. Contributions and challenges for network models a in cognitive neuroscience. Nat. Neurosci. 17, 652-660. doi doi: $10.1038 / \mathrm{nn} .3690$

Sporns, O., Chialvo, D.R., Kaiser, M., Hilgetag, C.C., 2004. Organization, development and function of complex brain networks. Trends Cogn. Sci. 8, 418-425. doi 10.1016/j.tics.2004.07.008

Tzourio-Mazoyer, N., Landeau, B., Papathanassiou, D., Crivello, F., Etard, O., Delcroix, N., Mazoyer, B., Joliot, M., 2002. Automated anatomical labeling of activations in SPM using a macroscopic anatomical parcellation of the MNI MRI single-subject brain. Neuroimage 15, 273-289. doi $10.1006 /$ nimg. 2001.0978

Van den Heuvel, M.P., Pol, H.E.H., 2010. Exploring the brain network: A review on resting-state fMRI functional connectivity. Eur.

11 Neuropsychopharmacol. 20, 519-534. doi 10.1016/j.euroneuro. 2010.03 .008

Wang, F., Li, T., Wang, X., Zhu, S., Ding, C., 2011. Community discovery using nonnegative matrix factorization. Data Min. Knowl. Discov. 22, 493-521. doi $10.1007 /$ s10618-010-0181-y

Wang, X., Tian, J., Li, X., Dai, J., Ai, L., 2004. Detecting brain activations by constrained non-negative matrix factorization from task-related BOLD fMRI, in: Medical Imaging 2004: Physiology,

1. Function, and Structure from Medical Images, pp. 675-682. doi 10. $1117 / 12.536186$

Wee, C.Y., Yap, P.T., Zhang, D., Wang, L., Shen, D., 2014. Groupconstrained sparse fMRI connectivity modeling for mild cognitive impairment identification. Brain Struct. Funct. 219, 641-656. doi 10.1007/s00429-013-0524-8

Wild, S., Curry, J., Dougherty, A., 2004. Improving non-negative matrix factorizations through structured initialization. Pattern Recognit. 37, 2217-2232. doi 10.1016/j.patcog.2004.02.013.

Wu, K., Taki, Y., Sato, K., Sassa, Y., Inoue, K., Goto, R., Okada, K., Kawashima, R., He, Y., Evans, A.C., Fukuda, H., 2011. The overlapping community structure of structural brain network in 1. young healthy individuals. PLoS ONE 6, e19608. doi 10.1371/ 
journal.pone.0019608

Xia, M., Wang, J., He, Y., 2013. BrainNet viewer: A network visualization tool for human brain connectomics. PloS ONE 8, e68910. doi 10.1371/journal.pone.0068910

Xie, J., Douglas, P.K., Wu, Y.N., Brody, A.L., Anderson, A.E., 2017. Decoding the encoding of functional brain networks: An fMRI classification comparison of non-negative matrix factorization (NMF), independent component analysis (ICA), and sparse coding algorithms. J. Neurosci. Method. 282, 81-94. doi 10.1016/j. jneumeth.2017.03.008

Yan, C., Zang, Y., 2010. DPARSF: A MATLAB toolbox for "pipeline" data analysis of resting-state fMRI. Front. Syst. Neurosci. 4. doi 10.3389/fnsys.2010.00013

Yeo, B.T., Krienen, F.M., Chee, M.W., Buckner, R.L., 2014. Estimates of segregation and overlap of functional connectivity networks in the human cerebral cortex. Neuroimage 88, 212-227. doi https://doi.org/10.1016/j.neuroimage.2013.10.046.

Yeo, B.T., Krienen, F.M., Sepulcre, J., Sabuncu, M.R., Lashkari, D., 1465 Hollinshead, M., Roffman, J.L., Smoller, J.W., Zöllei, L., Polimeni, J.R., Fischl, B., Liu, H., Buckner, R.L., 2011. The organization of the human cerebral cortex estimated by intrinsic functional

1. connectivity. J. Neurophysiol. 106, 1125-1165. doi 10.1152/jn 00338.2011

1470 Zanto, T.P., Gazzaley, A., 2013. Fronto-parietal network: Flexible

1] hub of cognitive control. Trends Cogn. Sci. 17, 602-603. doi 10 1016/j.tics.2013.10.001

Zuo, X.N., Anderson, J.S., Bellec, P., Birn, R.M., Biswal, B.B., Blautzik, J., Breitner, J.C., Buckner, R.L., Calhoun, V.D., Castellanos, F.X., et al., 2014. An open science resource for establishing reliability and reproducibility in functional connectomics. Sci. Data 1, 140049. doi 10.1038/sdata.2014.49

Zuo, X.N., Kelly, C., Adelstein, J.S., Klein, D.F., Castellanos, F.X., Milham, M.P., 2010. Reliable intrinsic connectivity networks: Test-retest evaluation using ICA and dual regression approach.

1 Neuroimage 49,2163-2177. doi $10.1016 / j \cdot$ neuroimage.2009.10 080 .

Zuo, X.N., Xing, X.X., 2014. Test-retest reliabilities of resting-state fMRI measurements in human brain functional connectomics: A systems neuroscience perspective. Neurosci. Biobehav. Rev. 45, 100-118. doi 10.1016/j.neubiorev. 2014.05.009 